\title{
Enantioselective Total Syntheses of Welwitindolinone A and Fischerindoles I and G
}

\author{
Phil S. Baran* and Jeremy M. Richter \\ Contribution from the Department of Chemistry, The Scripps Research Institute, 10550 North \\ Torrey Pines Road, La Jolla, California 92037
}

\section{Supporting Information}

General Procedures. All reactions were carried out under a nitrogen atmosphere with dry solvents under anhydrous conditions, unless otherwise noted. Dry tetrahydrofuran (THF), triethylamine (TEA), dichloromethane (DCM), methanol (MeOH), dimethylformamide (DMF), and benzene were obtained by passing commercially available pre-dried, oxygen-free formulations through activated alumina columns. Yields refer to chromatographically and spectroscopically ( ${ }^{1} \mathrm{H}$ NMR) homogeneous materials, unless otherwise stated. Reagents were purchased at the highest commercial quality and used without further purification, unless otherwise stated. Reactions were monitored by thin-layer chromatography (TLC) carried out on $0.25 \mathrm{~mm}$ E. Merck silica gel plates (60F-254) using UV light as visualizing agent and $p$ anisaldehyde in ethanol/aqueous $\mathrm{H}_{2} \mathrm{SO}_{4} / \mathrm{CH}_{3} \mathrm{CO}_{2} \mathrm{H}$ and heat as developing agents. NMR spectra were recorded on either a Bruker DRX 600, DRX 500 or an AMX 400 and calibrated using residual undeuterated solvent as an internal reference. The following abbreviations were used to explain the multiplicities: $\mathrm{s}=$ singlet, $\mathrm{d}=$ doublet, $\mathrm{t}=$ triplet, $\mathrm{q}=$ quartet, $\mathrm{m}=$ multiplet, $\mathrm{b}=$ broad. IR spectra were recorded on a Perkin-Elmer Spetrum BX spectrometer. High resolution mass spectra (HRMS) were recorded on an Agilent Mass spectrometer (at Scripps) using ESITOF (electrospray ionization-time of flight) or a ThermoFinnigan Mass spectrometer (at UCSD) using FAB (fast atom bombardment), or EI (electron impact). Low resolution mass spectra (LRMS) were recorded on an Agilent (at Scripps) or ThermoFinnigan Mass spectrometer (at 
UCSD) GC-MS. Melting points (m.p.) are uncorrected and were recorded on a Fisher-Johns 12144 melting point apparatus. Optical rotations were obtained on a Perkin-Elmer 431 Polarimeter. Note: optical rotations are provided for both the natural and unnatural series.

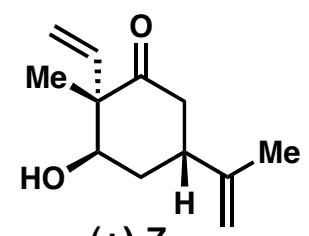

$(+)-7$

Compound 7: Magnesium turnings (14.6 g, $602 \mathrm{mmol}$ ) were flame dried in a round bottom flask equipped with a stir bar and addition funnel and allowed to cool under HIVAC. The magnesium turnings were then suspended in dry THF (120 mL) and the temperature of the flask controlled by a room-temperature water bath. Vinyl bromide solution (1.0 M, $120 \mathrm{~mL})$ was added dropwise over the course of 2 hours. Meanwhile, a flame-dried flask was charged with THF $(100 \mathrm{~mL})$ and cooled to $-78{ }^{\circ} \mathrm{C}$. Hexamethyldisilazane $(16.0 \mathrm{~mL}, 75.2 \mathrm{mmol})$ was added, followed by $n$-butyl lithium solution $(2.50 \mathrm{M}, 28.9 \mathrm{~mL})$. The resulting solution of LDA was then warmed to $0{ }^{\circ} \mathrm{C}$ for 30 minutes and cooled back to $-78{ }^{\circ} \mathrm{C}$, at which point a solution of carvone oxide $(10.0 \mathrm{~g}, 60.2 \mathrm{mmol})$ in $\mathrm{THF}$ $(60.2 \mathrm{~mL})$ was cannulated into the pre-cooled LDA solution. After stirring for 30 minutes, the enolate was warmed to $-15^{\circ} \mathrm{C}$ and the vinyl Grignard solution was cannulated into the enolate solution. The reaction was stirred for an additional 15 minutes then quenched with a saturated solution of $\mathrm{NH}_{4} \mathrm{Cl}(300 \mathrm{~mL})$, which was extracted three times with EtOAc $(300 \mathrm{~mL})$. The combined organic layers were washed with saturated $\mathrm{NaHCO}_{3}(200 \mathrm{~mL})$, water $(200 \mathrm{~mL})$, then brine $(200 \mathrm{~mL})$, then dried $\left(\mathrm{MgSO}_{4}\right)$, and the solvent removed by rotary evaporation. The crude reaction was purified by flash column chromatography (silica gel, 4:1 hexanes:EtOAc) to give 7 (3.51 g, 30\%); yellow oil; $\mathrm{R}_{\mathrm{f}}=0.25$ (silica gel, 3:1 hexanes:EtOAc); $[\alpha]_{\mathrm{D}}$ nat. $=-45.8\left(\mathrm{CH}_{2} \mathrm{Cl}_{2}\right.$, c 3.32), ent. $=+69.8\left(\mathrm{CH}_{2} \mathrm{Cl}_{2}, c\right.$ 0.56); IR (film) $v_{\max } 3453,2936,1702,1645,1420,1373,1107$, 1054, 926, $893 \mathrm{~cm}^{-1} ;{ }^{1} \mathrm{H}$ NMR (400 MHz, $\left.\mathrm{CDCl}_{3}\right) \delta 5.90(\mathrm{dd}, J=17.6,10.8 \mathrm{~Hz}, 1 \mathrm{H}), 5.15(\mathrm{~d}, J$ 
$=10.8 \mathrm{~Hz}, 1 \mathrm{H}), 4.97(\mathrm{~d}, J=17.6 \mathrm{~Hz}, 1 \mathrm{H}), 4.75(\mathrm{~s}, 1 \mathrm{H}), 4.71(\mathrm{~s}, 1 \mathrm{H}), 4.04(\mathrm{dd}, J=5.6,3.2 \mathrm{~Hz}$, $1 \mathrm{H}), 2.83-2.89(\mathrm{~m}, 1 \mathrm{H}), 2.57(\mathrm{t}, J=14.0 \mathrm{~Hz}, 1 \mathrm{H}), 2.32-2.37(\mathrm{~m}, 1 \mathrm{H}), 2.27(\mathrm{~d}, J=3.2 \mathrm{~Hz}, 1$ H), $2.00-2.07(\mathrm{~m}, 1 \mathrm{H}), 1.91-1.96(\mathrm{~m}, 1 \mathrm{H}), 1.71(\mathrm{~s}, 3 \mathrm{H}), 1.21(\mathrm{~s}, 3 \mathrm{H}) ;{ }^{13} \mathrm{C}$ NMR $(100 \mathrm{MHz}$, $\left.\mathrm{CDCl}_{3}\right) \delta$ 212.0, 147.2, 140.4, 116.5, 110.0, 76.5, 56.9, 43.2, 39.3, 33.5, 20.6, 19.4; HRMS (ESI) calcd. For $\mathrm{C}_{12} \mathrm{H}_{17} \mathrm{O}_{2}\left[\mathrm{M}-\mathrm{H}^{+}\right]$193.1234, found 193.1232.

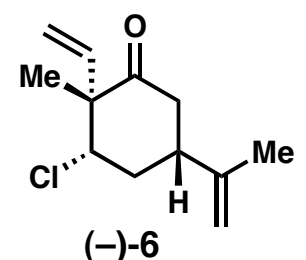

Compound 6: Compound 7 (2.47 g, $12.7 \mathrm{mmol})$ was dissolved in benzene. The solvent was then removed by rotary evaporation and the compound placed under HIVAC. Meanwhile, in a flame-dried flask, triphenyl phosphine $(3.33 \mathrm{~g}, 12.7 \mathrm{mmol})$ was dissolved in dry THF $(50 \mathrm{~mL})$. After dissolution, $N$ chlorosuccinimide $(1.70 \mathrm{~g}, 12.7 \mathrm{mmol})$ was added and the solution stirred for 30 minutes. Meanwhile, 7 was dissolved in dry THF $(50 \mathrm{~mL})$ and added to the previously mixed solution of triphenyl phosphine and NCS. The reaction was allowed to stir overnight at room temperature. In the morning, the solvent was removed by rotary evaporation and the residue dissolved in $\mathrm{CH}_{2} \mathrm{Cl}_{2}(200 \mathrm{~mL})$, washed three times with water $(150 \mathrm{~mL})$, once with brine $(150 \mathrm{~mL})$, and dried $\left(\mathrm{MgSO}_{4}\right)$. The solvent was removed by rotary evaporation and the crude material purified by flash column chromatography (silica gel, 5:1 hexane: $\mathrm{CH}_{2} \mathrm{Cl}_{2}$ ) to give pure $6(1.49 \mathrm{~g}, 55 \%)$; yellow oil; $\mathrm{R}_{\mathrm{f}}=0.62$ (silica gel, 1:1 hexanes: $\left.\mathrm{CH}_{2} \mathrm{Cl}_{2}\right) ;[\alpha]_{\mathrm{D}}$ nat. $=+45.6\left(\mathrm{CH}_{2} \mathrm{Cl}_{2}, c 0.55\right)$, ent. $=$ - $39.9\left(\mathrm{CH}_{2} \mathrm{Cl}_{2}, c\right.$ 0.67); IR (film) $v_{\max } 2977,1716,1647,1450,1374,1294,1226,1113,987$, 910, 860, $745 \mathrm{~cm}^{-1} ;{ }^{1} \mathrm{H}$ NMR $\left(400 \mathrm{MHz}, \mathrm{CDCl}_{3}\right) \delta 6.28(\mathrm{dd}, J=17.6,10.8 \mathrm{~Hz}, 1 \mathrm{H}), 5.27(\mathrm{~d}, J=$ $10.8 \mathrm{~Hz}, 1 \mathrm{H}), 5.03(\mathrm{~d}, J=17.6 \mathrm{~Hz}, 1 \mathrm{H}), 4.78(\mathrm{~s}, 1 \mathrm{H}), 4.72(\mathrm{~s}, 1 \mathrm{H}), 3.91(\mathrm{dd}, J=12.4,4.0 \mathrm{~Hz}$, $1 \mathrm{H}), 2.59(\mathrm{t}, J=13.6 \mathrm{~Hz}, 1 \mathrm{H}), 2.24-2.34(\mathrm{~m}, 3 \mathrm{H}), 2.10-2.16(\mathrm{~m}, 1 \mathrm{H}), 1.70(\mathrm{~s}, 3 \mathrm{H}), 1.32(\mathrm{~s}$, 
$3 \mathrm{H}) ;{ }^{13} \mathrm{C}$ NMR $\left(100 \mathrm{MHz}, \mathrm{CDCl}_{3}\right) \delta$ 207.3, 145.5, 136.6, 118.4, 110.8, 66.5, 57.9, 43.0, 42.0, 37.7, 21.1, 20.3; LRMS (GC-MS) calcd. for $\mathrm{C}_{12} \mathrm{H}_{18} \mathrm{ClO}\left[\mathrm{M}+\mathrm{H}^{+}\right]$213, found 213.

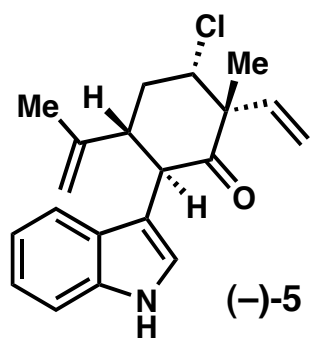

Compound 5: Compound 6 (1.29 g, $6.1 \mathrm{mmol})$ and indole (1.42 g, 12.2 mmol) were dissolved in benzene and the solvent removed by rotary evaporation. The resulting dried starting materials were placed on HIVAC cooled to $-78{ }^{\circ} \mathrm{C}$. Hexamethyldisilazane $(4.44 \mathrm{~mL}, 21.3 \mathrm{mmol})$ was added to the cooled THF, followed by $n$-butyl lithium solution $(7.54 \mathrm{~mL}, 18.8 \mathrm{mmol})$. The resulting solution of LDA was warmed to $0{ }^{\circ} \mathrm{C}$ for 30 minutes, then cooled back to $-78{ }^{\circ} \mathrm{C}$, at which point a solution of the starting materials in THF $(18.2 \mathrm{~mL})$ was added to the base. Stirring was continued for 30 minutes, at which time the septum was removed, copper(II)2-ethylhexanoate (3.19 g, $9.12 \mathrm{mmol})$ was rapidly added, and the septum quickly replaced. (Brief exposure of the reaction to atmospheric conditions has a trivial impact on the outcome and yield of the reaction). The solution was stirred for 10 minutes at $-78^{\circ} \mathrm{C}$ then warmed to room temperature. As soon as the flask achieved room temperature, the reaction was quenched by pouring into $1 \mathrm{~N} \mathrm{HCl}(100 \mathrm{~mL})$. This mixture was extracted three times with EtOAc $(100 \mathrm{~mL})$. The combined organic extracts were washed sequentially with $1 \mathrm{~N} \mathrm{NaOH}(100 \mathrm{~mL})$, water $(100 \mathrm{~mL})$, and brine $(100 \mathrm{~mL})$, then dried $\left(\mathrm{MgSO}_{4}\right)$, and the solvent removed by rotary evaporation. The crude reaction was purified by flash column chromatography (silica gel, gradient from 8:1 to 5:1 hexanes:EtOAc) to give 5 $(1.09 \mathrm{~g}, 55 \%)$; clear cubes; m.p. $=169-170{ }^{\circ} \mathrm{C} ; \mathrm{R}_{\mathrm{f}}=0.35$ (silica gel, 3:1 hexanes:EtOAc); $[\alpha]_{D}$ nat. $=+28.8\left(\mathrm{CH}_{2} \mathrm{Cl}_{2}, c 1.11\right)$, ent. $=-28.4\left(\mathrm{CH}_{2} \mathrm{Cl}_{2}, c\right.$ 1.07); IR (film) $v_{\max } 3414,1712,1584$, 1548, 1453, 991, 908, $740 \mathrm{~cm}^{-1}$; ${ }^{1} \mathrm{H}$ NMR $\left(500 \mathrm{MHz}, \mathrm{CDCl}_{3}\right) \delta 8.08$ (bs, $1 \mathrm{H}, \mathrm{D}_{2} \mathrm{O}$ exchangeable), $7.28(\mathrm{t}, J=7.5 \mathrm{~Hz}, 2 \mathrm{H}), 7.15(\mathrm{t}, J=7.0 \mathrm{~Hz}, 1 \mathrm{H}), 7.07(\mathrm{t}, J=7.0 \mathrm{~Hz}, 1 \mathrm{H}), 6.77$ 
$(\mathrm{d}, J=2.5 \mathrm{~Hz}, 1 \mathrm{H}), 6.61(\mathrm{dd}, J=18.0 \mathrm{~Hz}, 11.0 \mathrm{~Hz}, 1 \mathrm{H}), 5.50(\mathrm{~d}, J=10.5 \mathrm{~Hz}, 1 \mathrm{H}), 5.24(\mathrm{~d}, J=$ $17.5 \mathrm{~Hz}, 1 \mathrm{H}), 4.68(\mathrm{~s}, 1 \mathrm{H}), 4.63(\mathrm{~s}, 1 \mathrm{H}), 4.32(\mathrm{~d}, J=12.5 \mathrm{~Hz}, 1 \mathrm{H}), 4.15(\mathrm{dd}, J=12.5,4.5 \mathrm{~Hz}$, $1 \mathrm{H}), 2.86(\mathrm{td}, J=12.5,3.5 \mathrm{~Hz}, 1 \mathrm{H}), 2.55(\mathrm{q}, J=26.0,12.5 \mathrm{~Hz}, 1 \mathrm{H}), 2.36(\mathrm{dt}, J=13.5,4.0 \mathrm{~Hz}$, $1 \mathrm{H}), 1.56$ (s, $3 \mathrm{H}), 1.46$ (s, $3 \mathrm{H}) ;{ }^{13} \mathrm{C}$ NMR $\left(125 \mathrm{MHz}, \mathrm{CDCl}_{3}\right) \delta$ 207.1, 145.2, 138.0, 136.4, 127.7, 123.8, 122.2, 120.0, 119.1, 119.0, 113.8, 111.8, 110.9, 66.9, 58.9, 48.9, 48.4, 39.6, 22.7, 18.6; HRMS (ESI) calcd. For $\mathrm{C}_{20} \mathrm{H}_{23} \mathrm{ClNO}\left[\mathrm{M}+\mathrm{H}^{+}\right]$328.1463, found 328.1456. Structure verified by X-ray crystallography, see Figure 1.

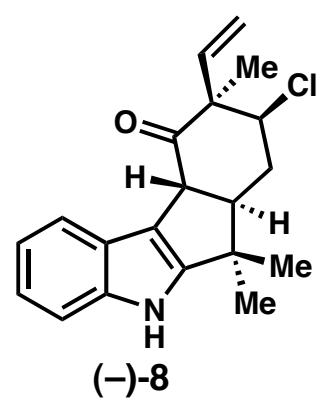

Compound 8: Compound $5(39.3 \mathrm{mg}, 0.12 \mathrm{mmol})$ was dissolved in 1,2dichloroethane $(7.0 \mathrm{~mL})$ and added to Montmorillonite $\mathrm{K}-10$ (1.57 g, $40 \mathrm{wt}$. equiv.) in a microwave reactor vessel. The slurry was irradiated at $120{ }^{\circ} \mathrm{C}$ for 6 minutes then cooled to room temperature. The slurry was diluted with EtOAc and the clay filtered off. The clay was further washed with EtOAc. The combined organic washings were evaporated under reduced pressure and the crude reaction was purified by flash column chromatography (silica gel, gradient from 3:1 to 1:1 hexane: $\left.\mathrm{CH}_{2} \mathrm{Cl}_{2}\right)$ to give $\mathbf{8}(10.1$ $\mathrm{mg}, 26 \%)$ plus recovered starting material $(21.5 \mathrm{mg}, 55 \%)$. The recovered starting material was then resubmitted to the same procedure. After recycling, the overall isolated yield of $\mathbf{8}$ was $40 \%$ plus recovered starting material (30\%). Many other acids were screened for this reaction including TFA, $\mathrm{MeSO}_{3} \mathrm{H}$, TMSOTf, $\mathrm{H}_{2} \mathrm{SO}_{4}$, heat, TsOH, $\mathrm{BF}_{3} \bullet \mathrm{OEt}$, $\mathrm{HCl}$, Triflamide, $\mathrm{NH}_{2} \mathrm{SO}_{3} \mathrm{H}$, $\mathrm{AcOH}, \mathrm{PPTS}, \mathrm{AlCl}_{3}$, and $\mathrm{AlBr}_{3}$, although none were as high yielding and clean as this procedure. The product was obtained as a light yellow foam; $\mathrm{R}_{\mathrm{f}}=0.56$ (silica gel, $\mathrm{CH}_{2} \mathrm{Cl}_{2}$ ); $[\alpha]_{\mathrm{D}}$ nat. $=+$ 179.2 $\left(\mathrm{CH}_{2} \mathrm{Cl}_{2}, c\right.$ 0.13), ent. $=-109.4\left(\mathrm{CH}_{2} \mathrm{Cl}_{2}, c\right.$ 0.31); IR (film) $v_{\max } 3395,2959,1718,1655$, 1448, 1297, 1052, 998, 926, 877, $738 \mathrm{~cm}^{-1} ;{ }^{1} \mathrm{H}$ NMR (400 MHz, $\left.\mathrm{CDCl}_{3}\right) \delta 7.96$ (bs, $1 \mathrm{H}, \mathrm{D}_{2} \mathrm{O}$ 
exchangeable), $7.68-7.70(\mathrm{~m}, 1 \mathrm{H}), 7.26-7.28(\mathrm{~m}, 1 \mathrm{H}), 7.11-7.15(\mathrm{~m}, 2 \mathrm{H}), 6.47(\mathrm{dd}, J=$ 17.6, $10.8 \mathrm{~Hz}, 1 \mathrm{H}), 5.38(\mathrm{~d}, J=10.8 \mathrm{~Hz}, 1 \mathrm{H}), 5.30(\mathrm{~d}, J=17.6 \mathrm{~Hz}, 1 \mathrm{H}), 4.10(\mathrm{~d}, J=12.0 \mathrm{~Hz}, 1$ H), $4.05(\mathrm{dd}, J=11.2,5.2 \mathrm{~Hz}, 1 \mathrm{H}), 2.24-2.41(\mathrm{~m}, 3 \mathrm{H}), 1.51(\mathrm{~s}, 3 \mathrm{H}), 1.40(\mathrm{~s}, 3 \mathrm{H}), 1.15(\mathrm{~s}, 3$ $\mathrm{H}) ;{ }^{13} \mathrm{C}$ NMR $\left(100 \mathrm{MHz}, \mathrm{CDCl}_{3}\right) \delta 205.6,150.8,139.7,137.5,124.1,121.3,120.3,120.1,118.2$, 112.5, 111.6, 68.0, 58.8, 53.5, 52.2, 41.2, 33.4, 25.0, 21.0, 20.3; HRMS (ESI) calcd. For $\mathrm{C}_{20} \mathrm{H}_{23} \mathrm{ClNO}\left[\mathrm{M}+\mathrm{H}^{+}\right]$328.1463, found 328.1470.

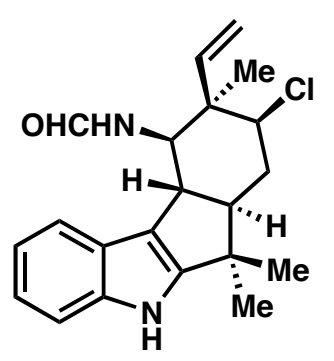

$(-)-11$

Compound 11: Ammonium acetate $(7.00 \mathrm{~g}, 91.5 \mathrm{mmol})$ and sodium cyanoborohydride ( $288 \mathrm{mg}, 4.58 \mathrm{mmol})$ were dissolved in methanol (100 $\mathrm{mL})$ at room temperature. Compound $8(750 \mathrm{mg}, 2.29 \mathrm{mmol})$ was dissolved in THF (20 mL) and added to the methanol solution. The reaction mixture was degassed by bubbling argon through the solution for 30 minutes, then it was stirred at room temperature for 7 days, with the addition of two more equivalents of sodium cyanoborohydride $(288 \mathrm{mg}$ ) and purging of the solution with argon on a daily basis. The reaction was then quenched with $2 \mathrm{~N} \mathrm{NaOH}(100 \mathrm{~mL})$ and extracted three times with EtOAc $(100 \mathrm{~mL})$. The combined organic extracts were washed with brine $(50 \mathrm{~mL})$, dried $\left(\mathrm{MgSO}_{4}\right)$, and evaporated under reduced pressure. The crude reaction was purified by flash column chromatography (silica gel, gradient from 1:1 hexane:EtOAc to 100\% EtOAc) to give 9 (198 mg, 18\%) plus recovered starting material (345 mg, 46\%). $\mathrm{R}_{\mathrm{f}}=0.38$ (silica gel, EtOAc). Amine 9 was then used directly for further reaction. The following compounds were added sequentially to a flask, maintained at room temperature, in the following order: compound 9 (132.3 mg, $0.40 \mathrm{mmol})$, formic acid $(15.1 \mu \mathrm{L}, 0.40 \mathrm{mmol})$, 2-chloro-4,6-dimethoxy-1,3,5triazine $(77.7 \mathrm{mg}, 0.44 \mathrm{mmol})$, DMAP $(1.5 \mathrm{mg}, 0.012 \mathrm{mmol}), N$-methylmorpholine $(49.8 \mu \mathrm{L}$, 
$0.44 \mathrm{mmol})$, and $\mathrm{CH}_{2} \mathrm{Cl}_{2}(1.1 \mathrm{~mL})$. The reaction was stirred for 45 minutes at room temperature, after which time the reaction was diluted with $\mathrm{CH}_{2} \mathrm{Cl}_{2}(75 \mathrm{~mL})$ and washed twice with $1 \mathrm{~N} \mathrm{HCl}$ $(50 \mathrm{~mL})$, once with saturated $\mathrm{NaHCO}_{3}(50 \mathrm{~mL})$, once with water $(50 \mathrm{~mL})$, and brine $(50 \mathrm{~mL})$. The organic layer was dried $\left(\mathrm{MgSO}_{4}\right)$ and evaporated under reduced pressure to give 11 (139.9 mg, 98\%), which was sufficiently pure for further reaction; light yellow oil; $\mathrm{R}_{\mathrm{f}}=0.46$ (silica gel, 1:1 hexane:EtOAc); $[\alpha]_{\mathrm{D}}$ nat. $=+20.0\left(\mathrm{CH}_{2} \mathrm{Cl}_{2}, c 0.20\right)$, ent. $=-11.8\left(\mathrm{CH}_{2} \mathrm{Cl}_{2}, c 0.72\right)$; IR (film) $v_{\max } 3394,3290,2960,1678,1570,1498,1476,1449,1365,1304,1273,1241,1205,1141$, 1104, 1072, 998, 909, $735 \mathrm{~cm}^{-1}$; ${ }^{1} \mathrm{H}$ NMR (400 MHz, $\left.\mathrm{CDCl}_{3}\right) \delta 8.50$ (s, $\left.1 \mathrm{H},\right), 7.95$ (bs, $1 \mathrm{H}, \mathrm{D}_{2} \mathrm{O}$ exchangeable), $7.34(\mathrm{~d}, J=7.2 \mathrm{~Hz}, 1 \mathrm{H}), 7.22(\mathrm{~d}, J=7.6 \mathrm{~Hz}, 1 \mathrm{H}), 7.08-7.14(\mathrm{~m}, 2 \mathrm{H}), 6.18(\mathrm{~d}$, $J=8.8 \mathrm{~Hz}, 1 \mathrm{H}) 5.84(\mathrm{dd}, J=17.2,10.8 \mathrm{~Hz}, 1 \mathrm{H}), 5.20(\mathrm{~d}, J=17.2 \mathrm{~Hz}, 1 \mathrm{H}), 5.12(\mathrm{~d}, J=12.0$ $\mathrm{Hz}, 1 \mathrm{H}), 4.35(\mathrm{dd}, J=9.2,5.6 \mathrm{~Hz}, 1 \mathrm{H}), 3.99(\mathrm{dd}, J=12.4,5.2 \mathrm{~Hz}, 2 \mathrm{H}), 3.86(\mathrm{t}, J=5.6 \mathrm{~Hz})$, $2.51-2.57(\mathrm{~m}, 1 \mathrm{H}), 2.05-2.18(\mathrm{~m}, 2 \mathrm{H}), 1.36(\mathrm{~s}, 3 \mathrm{H}), 1.31(\mathrm{~s}, 3 \mathrm{H}), 1.20(\mathrm{~s}, 3 \mathrm{H}) ;{ }^{13} \mathrm{C} \mathrm{NMR}$ $\left(100 \mathrm{MHz}, \mathrm{CDCl}_{3}\right) \delta 203.2,169.9,160.6,150.3,140.4,135.5,124.1,120.8,120.0,119.5,115.8$, $111.9,67.5,54.4,53.6,46.7,42.1,32.3,25.7,22.0,20.9$; HRMS (ESI) calcd. For $\mathrm{C}_{21} \mathrm{H}_{25} \mathrm{ClNaN}_{2} \mathrm{O}\left[\mathrm{M}+\mathrm{Na}^{+}\right] 356.1548$, found 379.1543 .

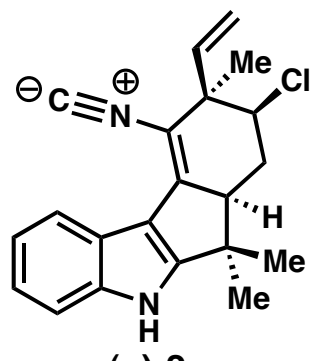

$(+)-2$

Fischerindole I: The freshly prepared $11(8.2 \mathrm{mg}, 0.023 \mathrm{mmol})$ was dissolved in THF $(0.60 \mathrm{~mL})$ and cooled to $0{ }^{\circ} \mathrm{C}$. An aluminum foil covering was placed around the flask to prevent light from entering the reaction. TEA $(3.1 \mu \mathrm{L}, 0.023 \mathrm{mmol})$ was added, followed by freshly prepared tert-butyl hypochlorite $(4.0 \mu \mathrm{L}, 0.034 \mathrm{mmol})$. After stirring for 10 minutes, the solvent was removed by rotary evaporation. The crude reaction was then purified by preparative thin layer chromatography (1:1 hexane:EtOAc, TEA treated plate). The compound was then 
dissolved in $\mathrm{CDCl}_{3}$, which proved to be crucial for successful completion of the reaction, to give 13a/b (7.3 mg, 89\%). Compound 13a/b was an unstable compound in solution and could not be fully characterized, it was reacted further immediately after preparation. Compound 13a/b (4.6 $\mathrm{mg}, 0.013 \mathrm{mmol})$ was dissolved in benzene $(1.0 \mathrm{~mL})$ and Burgess reagent $(6.2 \mathrm{mg}, 0.026 \mathrm{mmol})$ was added at room temperature. Upon completion of the reaction, as determined by TLC, the solvent was removed by rotary evaporation and the crude material purified by preparative thin layer chromatography (3:1 hexane:EtOAc), to give $2(2.3 \mathrm{mg}, 53 \%)$; pale yellow oil; $\mathrm{R}_{\mathrm{f}}=0.34$ (silica gel, 3:1 hexane:EtOAc); $[\alpha]_{\mathrm{D}}$ nat. $=-14.0\left(\mathrm{CH}_{2} \mathrm{Cl}_{2}, c 0.05\right)$, ent. $=+24.4\left(\mathrm{CH}_{2} \mathrm{Cl}_{2}, c\right.$ 0.09); IR (film) $v_{\max } 3272,2918,2099,1648,1474,1452,1259,1112,926,818,795,739,707$ $\mathrm{cm}^{-1}$; See comparison table and attached spectra for ${ }^{1} \mathrm{H}$ NMR data; HRMS (ESI) calcd. for $\mathrm{C}_{21} \mathrm{H}_{20} \mathrm{ClN}_{2}\left[\mathrm{M}-\mathrm{H}^{+}\right]$335.1320, found 335.1312.

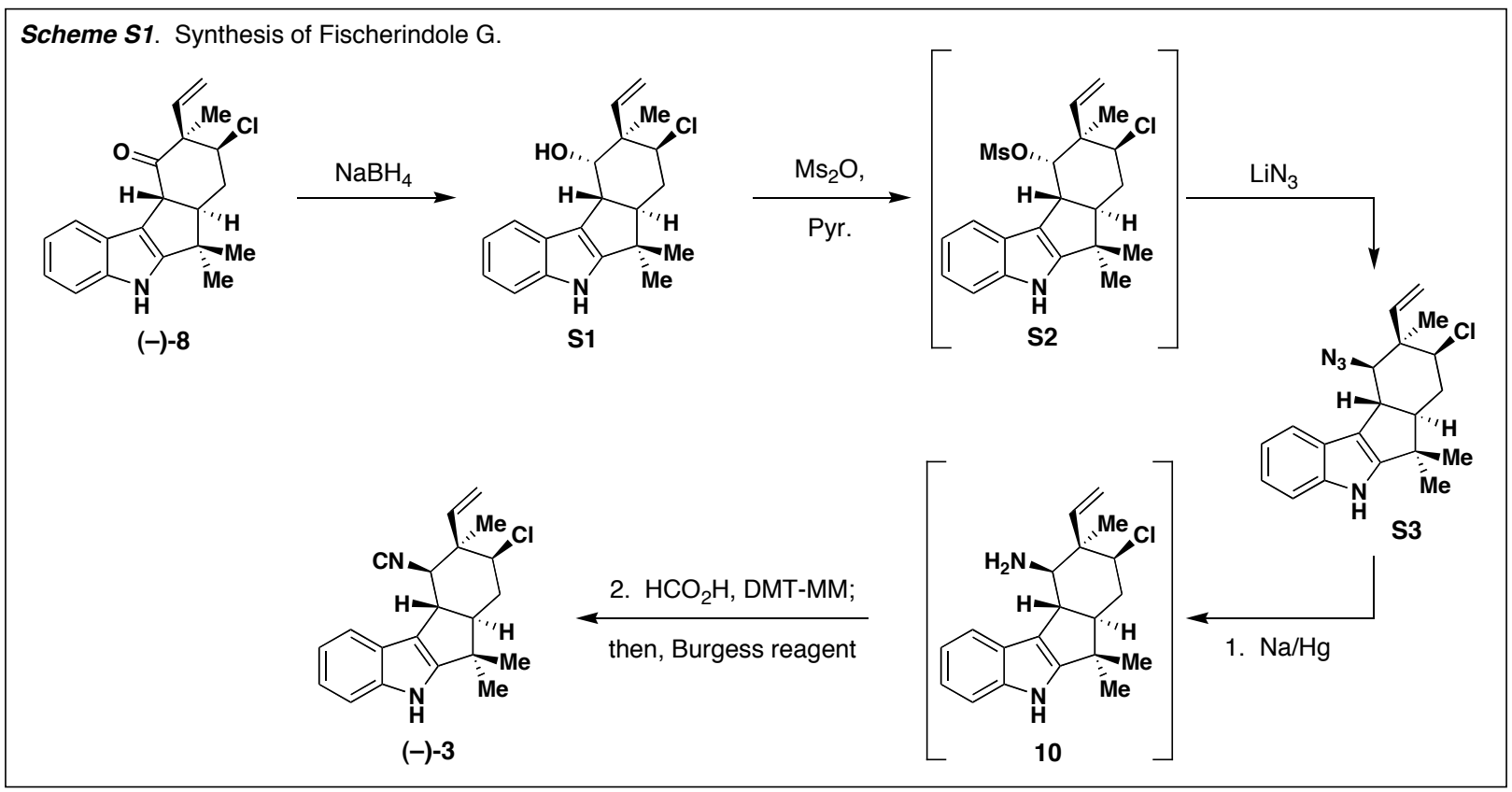




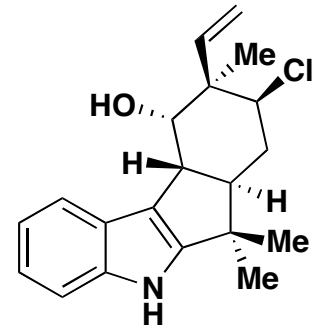

Compound S1: Compound 8 (78.9 mg, $0.241 \mathrm{mmol})$ was dissolved in methanol $(2.41 \mathrm{~mL})$ and cooled to $0{ }^{\circ} \mathrm{C}$. To this solution was added sodium borohydride $(8.9 \mathrm{mg}, 0.241 \mathrm{mmol})$ and stirring was continued for five minutes, after which time the reaction was quenched with saturated $\mathrm{NH}_{4} \mathrm{Cl}$

$(5 \mathrm{~mL})$. The reaction was diluted with EtOAc $(20 \mathrm{~mL})$ and washed further with water $(10 \mathrm{~mL})$ then brine $(10 \mathrm{~mL})$, then dried $\left(\mathrm{MgSO}_{4}\right)$ and the solvent removed by rotary evaporation. The crude $\mathbf{S 1}$ was sufficiently pure for further reaction $(79.0 \mathrm{mg}, 100 \%)$; yellow foam; $\mathrm{R}_{\mathrm{f}}=0.49$ (silica gel, $\left.\mathrm{CH}_{2} \mathrm{Cl}_{2}\right) ;[\alpha]_{\mathrm{D}}=-21.2\left(\mathrm{CH}_{2} \mathrm{Cl}_{2}, c 0.40\right)$; IR (film) $v_{\max } 3397,2984,2868,1455,1428$, 1300, 1191, 1104, 973, $745 \mathrm{~cm}^{-1} ;{ }^{1} \mathrm{H}$ NMR $\left(400 \mathrm{MHz}, \mathrm{CDCl}_{3}\right) \delta 7.84\left(\mathrm{~s}, 1 \mathrm{H}, \mathrm{D}_{2} \mathrm{O}\right.$ Exchangeable), $7.78(\mathrm{~d}, J=6.4 \mathrm{~Hz}, 1 \mathrm{H}), 7.31(\mathrm{dd}, J=6.4,1.6 \mathrm{~Hz}, 1 \mathrm{H}), 7.06-7.14(\mathrm{~m}, 2 \mathrm{H})$, $6.20(\mathrm{dd}, J=17.2,10.8 \mathrm{~Hz}, 1 \mathrm{H}), 5.55(\mathrm{~d}, J=11.2 \mathrm{~Hz}, 1 \mathrm{H}), 5.38(\mathrm{dd}, J=17.2,1.2 \mathrm{~Hz}, 1 \mathrm{H})$, $3.97(\mathrm{dd}, J=11.2,7.6 \mathrm{~Hz}, 1 \mathrm{H}), 3.44(\mathrm{t}, J=10.8 \mathrm{~Hz}, 1 \mathrm{H}), 2.82(\mathrm{t}, J=10.4 \mathrm{~Hz}, 1 \mathrm{H}), 1.94-2.20$ (m, $4 \mathrm{H}), 1.50(\mathrm{~s}, 3 \mathrm{H}), 1.39$ (s, $3 \mathrm{H}), 1.10(\mathrm{~s}, 3 \mathrm{H}) ;{ }^{13} \mathrm{C}$ APT NMR (100 MHz, $\left.\mathrm{CDCl}_{3}\right) \delta$ 151.1, $139.4,136.1,124.1,120.8,120.4,119.8,111.3,79.3,67.8,64.3,57.7,50.3,46.5,40.7,32.0$, 29.6, 25.1, 22.0, 20.3. HRMS (ESI) calcd. for $\mathrm{C}_{20} \mathrm{H}_{23} \mathrm{ClNO}\left[\mathrm{M}-\mathrm{H}^{+}\right]$328.1474, found 328.1484.

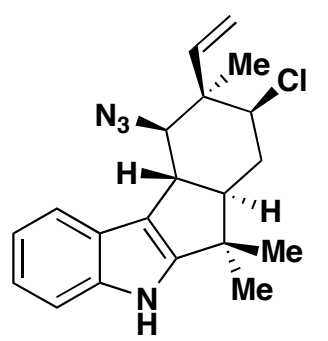

Compound S3: Compound S1 (17.1 mg, $0.052 \mathrm{mmol})$ was dissolved in pyridine $(0.515 \mathrm{~mL})$ and methanesulfonic anhydride $(18.0 \mathrm{mg}, 0.103 \mathrm{mmol})$ was added. The reaction was heated to $65{ }^{\circ} \mathrm{C}$ for 30 minutes then cooled back to room temperature. The reaction was diluted with diethyl ether (10 $\mathrm{mL})$ and washed twice with $1 \mathrm{~N} \mathrm{HCl}(10 \mathrm{~mL})$, water $(5 \mathrm{~mL})$, and brine $(5 \mathrm{~mL})$, then dried $\left(\mathrm{MgSO}_{4}\right)$ and the solvent removed by rotary evaporation. The crude reaction was purified by flash column chromatography (silica gel, gradient from 1:1 to $1: 2$ hexane: $\mathrm{CH}_{2} \mathrm{Cl}_{2}$ ), to give the 
mesylate $\mathbf{S 2}(15.2 \mathrm{mg}, 69 \%) . \quad \mathrm{R}_{\mathrm{f}}=0.35$ (silica gel, $\left.\mathrm{CH}_{2} \mathrm{Cl}_{2}\right)$. The mesylate $\mathbf{S 2}(134 \mathrm{mg}, 0.328$ mmol) was then dissolved in DMF (10 mL) and lithium azide solution (20\% in water, $0.50 \mathrm{~mL})$ was added. The reaction was warmed to $100{ }^{\circ} \mathrm{C}$ and heating continued for 2 days. After cooling to room temperature, the reaction was diluted with diethyl ether $(50 \mathrm{~mL})$ and washed three times with water $(25 \mathrm{~mL})$ then brine $(25 \mathrm{~mL})$. The organic layer was dried $\left(\mathrm{MgSO}_{4}\right)$ and the solvent removed by rotary evaporation. The crude reaction was purified by flash column chromatography (silica gel, gradient from 1:1 hexane: $\mathrm{CH}_{2} \mathrm{Cl}_{2}$ to $100 \% \mathrm{CH}_{2} \mathrm{Cl}_{2}$ ), to give $\mathbf{S 3}(64.7$ mg, 58\%); white powder; $\mathrm{R}_{\mathrm{f}}=0.54$ (silica gel, 3:1 hexane:EtOAc); $[\alpha]_{\mathrm{D}}=+14.4\left(\mathrm{CH}_{2} \mathrm{Cl}_{2}, c\right.$ 1.07); IR (film) $v_{\max } 2363,2250,1704,1613,1472,1380,1094,914,732 \mathrm{~cm}^{-1} ;{ }^{1} \mathrm{H}$ NMR $(600$ $\left.\mathrm{MHz}, \mathrm{CDCl}_{3}\right) \delta 7.85$ (bs, $1 \mathrm{H}, \mathrm{D}_{2} \mathrm{O}$ exchangeable), $7.44(\mathrm{~d}, J=6.6 \mathrm{~Hz}, 1 \mathrm{H}), 7.31(\mathrm{~d}, J=8.4 \mathrm{~Hz}$, $1 \mathrm{H}), 7.08-7.12(\mathrm{~m}, 2 \mathrm{H}), 6.42(\mathrm{dd}, J=17.4,10.8 \mathrm{~Hz}, 1 \mathrm{H}), 5.36-5.40(\mathrm{~m}, 2 \mathrm{H}), 4.96(\mathrm{~s}, 1 \mathrm{H})$, $4.26(\mathrm{dd}, J=11.4,4.8 \mathrm{~Hz}, 1 \mathrm{H}), 3.56(\mathrm{dd}, J=10.8,2.4 \mathrm{~Hz}, 1 \mathrm{H}), 2.68(\mathrm{dt}, J=13.2,3.0 \mathrm{~Hz}, 1 \mathrm{H})$, $2.10-2.17(\mathrm{~m}, 2 \mathrm{H}), 1.51(\mathrm{~s}, 3 \mathrm{H}), 1.41(\mathrm{~s}, 3 \mathrm{H}), 1.11(\mathrm{~s}, 3 \mathrm{H}) ;{ }^{13} \mathrm{C}$ APT NMR (150 MHz, $\left.\mathrm{CDCl}_{3}\right) \delta 151.5,140.3,139.7,123.9,121.2,120.3,118.5,116.9,115.9,112.1,71.2,65.1,54.3$, 47.6, 44.0, 40.7, 33.0, 25.3 (2 carbons missing). LRMS (GC-MS) calcd. for $\mathrm{C}_{21} \mathrm{H}_{23} \mathrm{~N}_{4} \mathrm{Cl}[\mathrm{M}+$ $\left.\mathrm{H}^{+}\right]$355, found 355 .

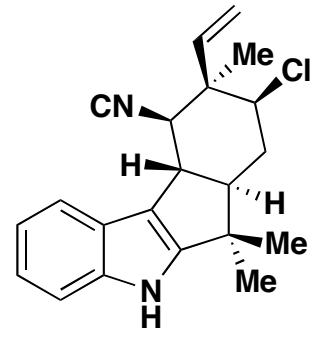

$(-)-3$

Fischerindole G: Compound S3 (47.4 mg, $0.133 \mathrm{mmol})$ was dissolved in ethanol (1.33 $\mathrm{mL})$ and sodium-mercury amalgum (266 mg, $1.33 \mathrm{mmol})$ was added. The reaction was heated to reflux for 2 hours then cooled to room temperature. The reaction was diluted with EtOAc $(25 \mathrm{~mL})$ and washed three times with water $(15 \mathrm{~mL})$ then brine $(15 \mathrm{~mL})$. The organic layer was dried $\left(\mathrm{MgSO}_{4}\right)$ and the solvent removed by rotary evaporation. The crude reaction was purified 
by flash column chromatography (silica gel, gradient from 5:1 to 3:1 hexane:EtOAc), to give $\mathbf{1 0}$ (29.2 mg, 66\%), which was then reacted further. $R_{f}=0.20$ (silica gel, EtOAc). The following compounds were added sequentially to a flask, maintained at room temperature, in the following order: Compound 10 (29.2 mg, $0.088 \mathrm{mmol})$, formic acid (3.6 $\mu \mathrm{L}, 0.097 \mathrm{mmol}), 2$-chloro-4,6dimethoxy-1,3,5-triazine (18.6 mg, $0.106 \mathrm{mmol})$, DMAP $(0.3 \mathrm{mg}, 0.003 \mathrm{mmol}), N$ methylmorpholine $(11.6 \mu \mathrm{L}, 0.106 \mathrm{mmol})$, and $\mathrm{CH}_{2} \mathrm{Cl}_{2}(0.232 \mathrm{~mL})$. The reaction was stirred for 2 hours at room temperature, after which time the reaction was diluted with $\mathrm{CH}_{2} \mathrm{Cl}_{2}(25 \mathrm{~mL})$ and washed twice with $1 \mathrm{~N} \mathrm{HCl}(15 \mathrm{~mL})$, once with saturated $\mathrm{NaHCO}_{3}(15 \mathrm{~mL})$, once with water $(15$ $\mathrm{mL})$, and brine $(15 \mathrm{~mL})$. The organic layer was dried $\left(\mathrm{MgSO}_{4}\right)$ and evaporated under reduced pressure. The crude reaction was purified by flash column chromatography (silica gel, gradient from 3:1 to 1:1 hexane:EtOAc) to give the corresponding formamide (27.4 mg, 87\%), $1.8 \mathrm{mg}$ of which $(0.0053 \mathrm{mmol})$ was dissolved in benzene $(0.50 \mathrm{~mL})$ and Burgess reagent $(5.0 \mathrm{mg}, 0.021$ mmol) was added at room temperature. Upon completion of the reaction, as determined by TLC, the solvent was removed by rotary evaporation and the crude material purified by preparative thin layer chromatography (3:1 hexane:EtOAc), to give $3(1.4 \mathrm{mg}, 82 \%)$; white powder; $\mathrm{R}_{\mathrm{f}}=$ 0.48 (silica gel, 3:1 hexane:EtOAc); $[\alpha]_{\mathrm{D}}=-50.0\left(\mathrm{CH}_{2} \mathrm{Cl}_{2}, c\right.$ 0.02); IR (film) $v_{\max } 3302,2954$, $2123,1716,1668,1456,1364,1127,1065,934,801,742$; See comparison table and attached spectra for ${ }^{1} \mathrm{H}$ NMR data; HRMS (EI) calcd. for $\mathrm{C}_{21} \mathrm{H}_{23} \mathrm{~N}_{2} \mathrm{Cl}\left[\mathrm{M}+\mathrm{H}^{+}\right] 338.1544$, found 338.1561 . 


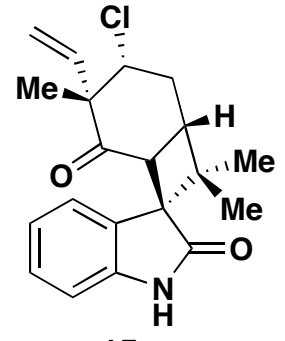

15

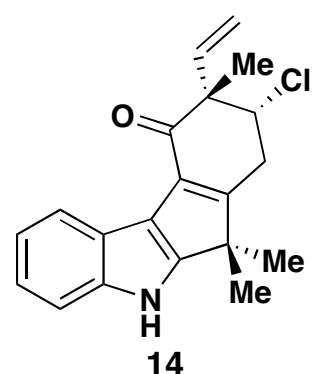

Compounds 15 and 14: Compound $8(13.1 \mathrm{mg}$, $0.040 \mathrm{mmol})$ was dissolved in THF $(0.40 \mathrm{~mL})$ and cooled to $0{ }^{\circ} \mathrm{C}$. Triethyl amine $(5.5 \mu \mathrm{L}, 0.040 \mathrm{mmol})$ and then $t$ - $\mathrm{BuOCl}(8.0 \mu \mathrm{L}, 0.068 \mathrm{mmol})$ was added.

The reaction was stirred for 10 minutes then the solvent was removed by rotary evaporation.

The crude residue was then dissolved in a 40:20:1 mixture of $\mathrm{MeOH}: \mathrm{H}_{2} \mathrm{O}: \mathrm{AcOH}(0.50 \mathrm{~mL})$ with gentle heating, and stirred for 10 minutes. The reaction was then quenched with saturated $\mathrm{NaHCO}_{3}(5 \mathrm{~mL})$ and extracted with EtOAc $(10 \mathrm{~mL})$. The organic layer was further washed with $\mathrm{H}_{2} \mathrm{O}(5 \mathrm{~mL})$ then brine $(5 \mathrm{~mL})$, dried $\left(\mathrm{MgSO}_{4}\right)$, and evaporated en vacuo. The crude reaction mixture was purified by flash column chromatography (silica gel, gradient from $8: 1$ to $3: 1$ hexanes:EtOAc) to give 15 (2.7 mg, 20\%) and 14 (2.7 mg, 21\%). Compound 15: clear needles; $\mathrm{R}_{\mathrm{f}}=0.18$ (silica gel, 3:1 hexane:EtOAc); $[\alpha]_{\mathrm{D}}=+84.7\left(\mathrm{CH}_{2} \mathrm{Cl}_{2}, c 0.64\right) ; \mathrm{IR}\left(\right.$ film) $v_{\max } 3371$, 2962, 2927, 2869, 1704, 1621, 1471, 1372, 1330, 1266, 1195, 1115, 926, 740; ${ }^{1} \mathrm{H}$ NMR (600 $\left.\mathrm{MHz}, \mathrm{CDCl}_{3}\right) \delta 7.79\left(\mathrm{bs}, 1 \mathrm{H}, \mathrm{D}_{2} \mathrm{O}\right.$ exchangeable), $7.17-7.21(\mathrm{~m}, 2 \mathrm{H}), 6.98(\mathrm{t}, J=7.2 \mathrm{~Hz}, 1 \mathrm{H})$, $6.84(\mathrm{~d}, J=7.8 \mathrm{~Hz}, 1 \mathrm{H}), 6.36(\mathrm{dd}, J=17.4,10.8 \mathrm{~Hz}, 1 \mathrm{H}), 5.26(\mathrm{~d}, J=10.8 \mathrm{~Hz}, 1 \mathrm{H}), 5.02(\mathrm{~d}, J$ $=17.4 \mathrm{~Hz}, 1 \mathrm{H}), 4.15(\mathrm{dd}, J=11.4,4.8 \mathrm{~Hz}, 1 \mathrm{H}), 3.43(\mathrm{~d}, J=13.8 \mathrm{~Hz}, 1 \mathrm{H}), 3.12(\mathrm{t}, J=12 \mathrm{~Hz}, 1$ $\mathrm{H}), 2.31-2.34(\mathrm{~m}, 1 \mathrm{H}), 2.10-2.17(\mathrm{~m}, 1 \mathrm{H}), 1.39(\mathrm{~s}, 3 \mathrm{H}), 1.35(\mathrm{~s}, 3 \mathrm{H}), 1.15(\mathrm{~s}, 3 \mathrm{H}) ;{ }^{13} \mathrm{C}$ APT NMR (150 MHz, CDCl3) $\delta 199.8,177.8,141.4,137.9,128.8,127.0,124.6,122.0,118.2$, 109.9, 68.4, 59.4, 50.9, 49.1, 44.6, 36.2, 23.5, 21.2, 20.1 (1 carbon missing); HRMS (ESI) calcd. for $\mathrm{C}_{20} \mathrm{H}_{23} \mathrm{ClNO}_{2}\left[\mathrm{M}+\mathrm{H}^{+}\right]$344.1412, found 344.1409. Compound 14: yellow oil; $\mathrm{R}_{\mathrm{f}}=0.31$ (silica gel, 3:1 hexane:EtOAc); $[\alpha]_{\mathrm{D}}=-10.3\left(\mathrm{CH}_{2} \mathrm{Cl}_{2}, c\right.$ 0.29); IR (film) $v_{\max } 3391,2927,1661$, 1480, 1446, 1418, 1264, 737; ${ }^{1} \mathrm{H}$ NMR (500 MHz, $\left.\mathrm{CDCl}_{3}\right) \delta 8.25$ (bs, $1 \mathrm{H}, \mathrm{D}_{2} \mathrm{O}$ exchangeable), $8.20(\mathrm{~d}, J=7.5 \mathrm{~Hz}, 1 \mathrm{H}), 7.35(\mathrm{~d}, J=7.0 \mathrm{~Hz}, 1 \mathrm{H}), 7.14-7.20(\mathrm{~m}, 2 \mathrm{H}), 6.16(\mathrm{dd}, J=17.5,11.0$ 
$\mathrm{Hz}, 1 \mathrm{H}), 5.25(\mathrm{~d}, J=10.5 \mathrm{~Hz}, 1 \mathrm{H}), 5.17(\mathrm{~d}, J=17.5 \mathrm{~Hz}, 1 \mathrm{H}), 4.39(\mathrm{dd}, J=9.5,5.0 \mathrm{~Hz}, 1 \mathrm{H})$, $2.90-3.01(\mathrm{~m}, 2 \mathrm{H}), 1.55(\mathrm{~s}, 3 \mathrm{H}), 1.40(\mathrm{~s}, 3 \mathrm{H}), 1.36(\mathrm{~s}, 3 \mathrm{H}) ;{ }^{13} \mathrm{C}$ APT NMR $(150 \mathrm{MHz}$, CDCl3) $\delta 195.0,163.1,153.5,140.7,135.9,134.4,122.7,122.5,121.5,118.9,118.7,116.6$, 112.4, 65.3, 61.3, 47.1, 32.1, 23.4, 23.3, 21.2; HRMS (ESI) calcd. for $\mathrm{C}_{20} \mathrm{H}_{21} \mathrm{CINO}\left[\mathrm{M}+\mathrm{H}^{+}\right]$ 326.1306, found 326.1303 .

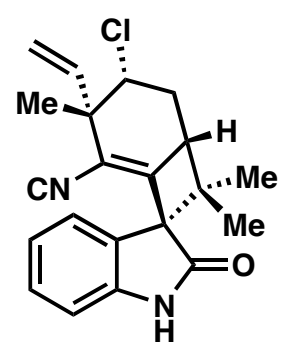

Welwitindolinone A (1): Fischerindole I (1.7 mg, $0.00479 \mathrm{mmol})$ ) was dissolved in THF $(0.040 \mathrm{~mL})$ and cooled to $-30{ }^{\circ} \mathrm{C}$ in a flask covered with aluminum foil. TEA $(0.66 \mu \mathrm{L}, 0.00479 \mathrm{mmol})$ was then added, followed by freshly prepared $t$ - $\mathrm{BuOCl}(0.79 \mu \mathrm{L}, 0.00719 \mathrm{mmol})$ and stirring was continued

for 1 minute. The reaction mixture was then evaporated under reduced pressure (HIVAC) while at $-30^{\circ} \mathrm{C}$. When all the volatile material was removed from the flask, the crude material was then dissolved in a mixture of 95:4:1 THF: $\mathrm{H}_{2} \mathrm{O}$ :TFA $(0.1 \mathrm{~mL})$ at $-30{ }^{\circ} \mathrm{C}$. The reaction was stirred for 5 minutes after which it was diluted with EtOAc $(2.0 \mathrm{~mL})$ and quenched with $5 \%$ $\mathrm{NaHCO}_{3}(1 \mathrm{~mL})$. The organic layer was further washed with brine $(1 \mathrm{~mL})$, dried $\left(\mathrm{Na}_{2} \mathrm{SO}_{4}\right)$, and the solvent removed by rotary evaporation. The crude material was purified by preparative thin layer chromatography (silica gel, 10:1 DCM:isooctane) to give 1 (0.4 mg, 25\%) and 3-epi-1 $(0.05 \mathrm{mg}, 3 \%)$, ratio as determined by ${ }^{1} \mathrm{H}$ NMR. Welwitindolinone A: pale green oil; $\mathrm{R}_{\mathrm{f}}=0.09$ (silica gel, 10:1 $\mathrm{CH}_{2} \mathrm{Cl}_{2}:$ isooctane); $[\alpha]_{\mathrm{D}}=+180\left(\mathrm{CH}_{2} \mathrm{Cl}_{2}, c\right.$ 0.0025); IR (film) $v_{\max } 3424,2075$, 1695, 1625, 1468, 1261, 1092; see following table and attached spectra for ${ }^{1} \mathrm{H}$ NMR; MS (DIOS) natural: $m / z$ 352/354 (3:1 $\mathrm{M}^{+}$ion cluster), $317\left(\mathrm{M}^{+}-\mathrm{Cl}\right)$, synthetic: $m / z 353 / 355\left(3: 1 \mathrm{M}^{+} \mathrm{H}^{+}\right)$, $317\left(\mathrm{M}^{+}-\mathrm{Cl}\right)$. HRMS (EI) calcd. for $\mathrm{C}_{21} \mathrm{H}_{21} \mathrm{ON}_{2} \mathrm{Cl}\left[\mathrm{M}+\mathrm{H}^{+}\right]$352.1337, found 352.1333. 3-epi1: light yellow oil; $\mathrm{R}_{\mathrm{f}}=0.17\left(10: 1 \mathrm{CH}_{2} \mathrm{Cl}_{2}\right.$ :isooctane); $[\alpha]_{\mathrm{D}}=+17.5(\mathrm{CH} 2 \mathrm{Cl} 2, c 0.08) ; \mathrm{IR}$ (film) 
$v_{\max } 3427,2070,1700,1620,1468,1110 ;{ }^{1} \mathrm{H}$ NMR $\left(500 \mathrm{MHz}, \mathrm{CD}_{2} \mathrm{Cl}_{2}\right) \delta 7.37$ (bs, $1 \mathrm{H}, \mathrm{D}_{2} \mathrm{O}$ exchangeable), $7.21(\mathrm{t}, J=8.0 \mathrm{~Hz}, 1 \mathrm{H}), 7.00(\mathrm{t}, J=7.5 \mathrm{~Hz}, 1 \mathrm{H}), 6.92(\mathrm{~d}, J=7.5 \mathrm{~Hz}, 1 \mathrm{H}), 6.83$ $(\mathrm{d}, J=8.0 \mathrm{~Hz}, 1 \mathrm{H}), 6.07$ (dd, $J=17.5,11.0 \mathrm{~Hz}, 1 \mathrm{H}), 5.40(\mathrm{~d}, J=11.0 \mathrm{~Hz}, 1 \mathrm{H}), 5.26(\mathrm{~d}$, buried under residual solvent, $1 \mathrm{H}), 4.11(\mathrm{dd}, J=11.0,5.0 \mathrm{~Hz}, 1 \mathrm{H}), 3.62(\mathrm{t}, J=8.0 \mathrm{~Hz}, 1 \mathrm{H}), 1.96-$ $1.99(\mathrm{~m}, 2 \mathrm{H}), 1.22(\mathrm{~s}, 3 \mathrm{H}), 1.18(\mathrm{~s}, 3 \mathrm{H}), 1.15$ (s, $3 \mathrm{H})$; HRMS (EI) calcd. for $\mathrm{C}_{21} \mathrm{H}_{21} \mathrm{ON}_{2} \mathrm{Cl}$ [M $\left.+\mathrm{H}^{+}\right]$352.1337, found 352.1334 . 
NOTE: Side-by-side spectral comparison at the end of supporting information

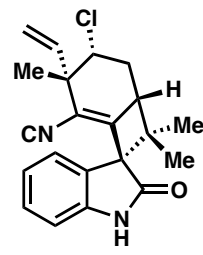

$(+)$-welwitindolinone A (1)

Reported

\begin{tabular}{c} 
Reported \\
\hline $1.19 \mathrm{~s}, 3 \mathrm{H}$ \\
$1.26 \mathrm{~s}, 3 \mathrm{H}$ \\
$1.43 \mathrm{~s}, 3 \mathrm{H}$ \\
$1.92 \mathrm{td}, 12.5 / 10.6,1 \mathrm{H}$ \\
$2.03 \mathrm{ddd}, 12.5 / 7.0 / 3.2,1 \mathrm{H}$ \\
$3.60 \mathrm{dd}, 10.6 / 7.0,1 \mathrm{H}$ \\
$4.04 \mathrm{dd}, 12.5 / 3.2,1 \mathrm{H}$ \\
$5.32 \mathrm{dd}, 17.3 / 0.5,1 \mathrm{H}$ \\
$5.46 \mathrm{dt}, 10.8 / 0.5,1 \mathrm{H}$ \\
$5.99 \mathrm{dd}, 17.3 / 10.8,1 \mathrm{H}$ \\
$6.92 \mathrm{ddd}, 7.7 / 1.2 / 0.6,1 \mathrm{H}$ \\
$7.06 \mathrm{td}, 7.7 / 1.2,1 \mathrm{H}$ \\
$7.24 \mathrm{ddd}, 7.7 / 1.2 / 0.6,1 \mathrm{H}$ \\
$7.29 \mathrm{td}, 7.7 / 1.2,1 \mathrm{H}$ \\
$7.62,7.50$ (two values \\
given) brs $(\mathrm{N}-\mathrm{H})$
\end{tabular}

Observed

\begin{tabular}{|c}
$1.19 \mathrm{~s}, 3 \mathrm{H}$ \\
$1.26 \mathrm{~s}, 3 \mathrm{H}$ \\
$1.43 \mathrm{~s}, 3 \mathrm{H}$ \\
$1.92 \mathrm{dd}, 22.8 / 12.0,1 \mathrm{H}^{*}$ \\
$2.03 \mathrm{~m}, 1 \mathrm{H}^{*}$ \\
$3.60 \mathrm{dd}, 10.8 / 7.2,1 \mathrm{H}$ \\
$4.04 \mathrm{dd}, 12.6 / 3.0,1 \mathrm{H}$ \\
$5.32 \mathrm{~d}, 13.8,1 \mathrm{H}^{*}$ \\
$5.46 \mathrm{~d}, 10.8,1 \mathrm{H}^{*}$ \\
$5.95 \mathrm{dd}, 17.4 / 10.8,1 \mathrm{H}$ \\
$6.92 \mathrm{~d}, 7.8,1 \mathrm{H}^{*}$ \\
$7.06 \mathrm{t}, 7.8,1 \mathrm{H}^{*}$ \\
$7.24 \mathrm{~d}, 7.8,1 \mathrm{H}^{*}$ \\
$7.29 \mathrm{t}, 7.2,1 \mathrm{H}^{*}$ \\
$7.41 \mathrm{brs}(\mathrm{N}-\mathrm{H})$
\end{tabular}

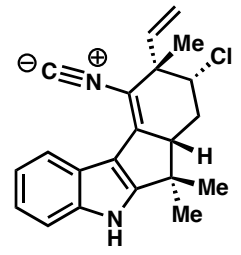

(-)-fischerindole I (2)

\begin{tabular}{c|c} 
Reported $^{1}$ & Observed \\
\hline $1.17 \mathrm{~s}, 3 \mathrm{H}$ & $1.17 \mathrm{~s}, 3 \mathrm{H}$ \\
$1.45 \mathrm{~s}, 3 \mathrm{H}$ & $1.44 \mathrm{~s}, 3 \mathrm{H}$ \\
$1.58 \mathrm{~s}, 3 \mathrm{H}$ & $1.58 \mathrm{~s}, 3 \mathrm{H}$ \\
$2.10 \mathrm{~m}, 2 \mathrm{H}$ & $2.09 \mathrm{~m}, 2 \mathrm{H}$ \\
$3.12 \mathrm{ddd}, 1 \mathrm{H}$ & $3.11 \mathrm{dd}, 11.0 / 8.5,1 \mathrm{H}^{*}$ \\
$4.14 \mathrm{~m}, 1 \mathrm{H}$ & $4.13 \mathrm{~m}, 1 \mathrm{H}$ \\
$5.14 \mathrm{~d}, 1 \mathrm{H}$ & $5.14 \mathrm{~d}, 17.5,1 \mathrm{H}$ \\
$5.34 \mathrm{~d}, 1 \mathrm{H}$ & $5.33 \mathrm{~d}, 13.0,1 \mathrm{H}$ \\
$5.89 \mathrm{dd}, 1 \mathrm{H}$ & $5.89 \mathrm{dd}, 17.5 / 10.5,1 \mathrm{H}$ \\
$7.18 \mathrm{~m}, 2 \mathrm{H}$ & $7.18 \mathrm{~m}, 2 \mathrm{H}$ \\
$7.38 \mathrm{~d}, 1 \mathrm{H}$ & $7.38 \mathrm{~d}, 7.5,1 \mathrm{H}$ \\
$8.29 \mathrm{dd}, 1 \mathrm{H}$ & $8.29 \mathrm{~d}, 8.0,1 \mathrm{H}$ \\
$8.38,8.40$ (two & $8.37, \mathrm{brs}(\mathrm{N}-\mathrm{H})$ \\
values given) & \\
brs (N-H) &
\end{tabular}

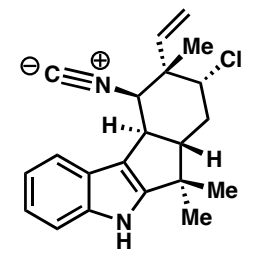

(+)-fischerindole G (3)

Reported Observed

\begin{tabular}{c|c}
\multicolumn{1}{c}{ Reported } & Observed \\
\hline $1.11 \mathrm{~s}, 3 \mathrm{H}$ & $1.11 \mathrm{~s}, 3 \mathrm{H}$ \\
$1.44 \mathrm{~s}, 3 \mathrm{H}$ & $1.44 \mathrm{~s}, 3 \mathrm{H}$ \\
$1.55 \mathrm{~s}, 3 \mathrm{H}$ & $1.54 \mathrm{~s}, 3 \mathrm{H}$ \\
$2.08 \mathrm{q}, 13.4 / 12.4,2 \mathrm{H}$ & $2.08 \mathrm{q}, 13.0 / 12.0,2 \mathrm{H}$ \\
$2.19 \mathrm{ddd}, 12.5 / 4.7 / 2.9,1 \mathrm{H}$ & $2.19 \mathrm{dd}, 12.5 / 3.5,1 \mathrm{H}$ \\
$2.45 \mathrm{ddd}, 13.4 / 10.5 / 2.9,1 \mathrm{H}$ & $2.45 \mathrm{td}, 13.5 / 11.0,1 \mathrm{H}^{*}$ \\
$3.30 \mathrm{dtd}, 10.5 / 2.7,1 \mathrm{H}$ & $3.32 \mathrm{~m}, 1 \mathrm{H}^{*}$ \\
$4.18 \mathrm{dd}, 12.0 / 4.7,1 \mathrm{H}$ & $4.18 \mathrm{dd}, 12.0 / 4.5,1 \mathrm{H}$ \\
$4.53 \mathrm{~d}, 2.7,1 \mathrm{H}$ & $4.53 \mathrm{~d}, 2.5,1 \mathrm{H}$ \\
$5.35 \mathrm{~d}, 18.0,1 \mathrm{H}$ & $5.33 \mathrm{~d}, 19.5,1 \mathrm{H}$ \\
$5.39 \mathrm{~d}, 10.9,1 \mathrm{H}$ & $5.39 \mathrm{~d}, 11.5,1 \mathrm{H}$ \\
$6.30 \mathrm{dd}, 18.0 / 10.9,1 \mathrm{H}$ & $6.30 \mathrm{dd}, 17.5 / 11.0,1 \mathrm{H}$ \\
$7.07 \mathrm{ddd}, 7.7 / 7.7 / 1.1,1 \mathrm{H}$ & $7.07 \mathrm{~m}, 1 \mathrm{H}^{*}$ \\
$7.11 \mathrm{ddd}, 7.7 / 7.7 / 1.1,1 \mathrm{H}$ & $7.11 \mathrm{~m}, 1 \mathrm{H}^{*}$ \\
$7.36 \mathrm{dd}, 7.7 / 1.1,1 \mathrm{H}$ & $7.36 \mathrm{~d}, 7.5,1 \mathrm{H}^{*}$ \\
$7.41 \mathrm{dd}, 7.7 / 1.1,1 \mathrm{H}$ & $7.41 \mathrm{~d}, 7.5,1 \mathrm{H}^{*}$ \\
$8.06 \mathrm{brs}(\mathrm{N}-\mathrm{H})$ & $8.05 \mathrm{brs}(\mathrm{N}-\mathrm{H})$ \\
&
\end{tabular}

${ }^{1}$ From the footnote on table 2 in J. Am. Chem. Soc. 1994, 116, 9935: "the coupling constants for [Fischerindole I] are within $\pm 0.5 \mathrm{~Hz}$ of the values for [Fischerindole G]".

*Further splitting was not distinguishable on our spectrometers (nor on Moore's it would appear): see the spectra. 


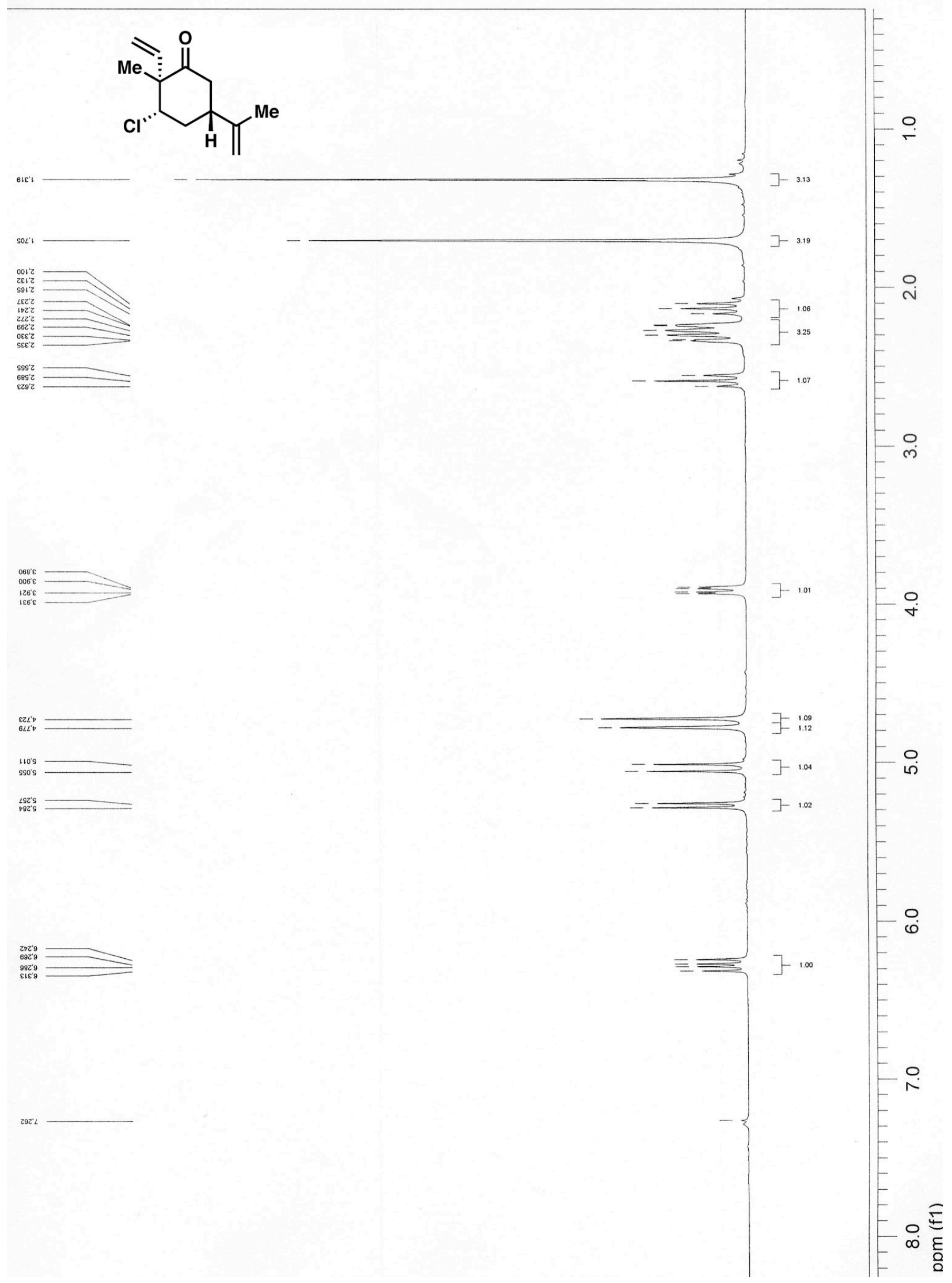




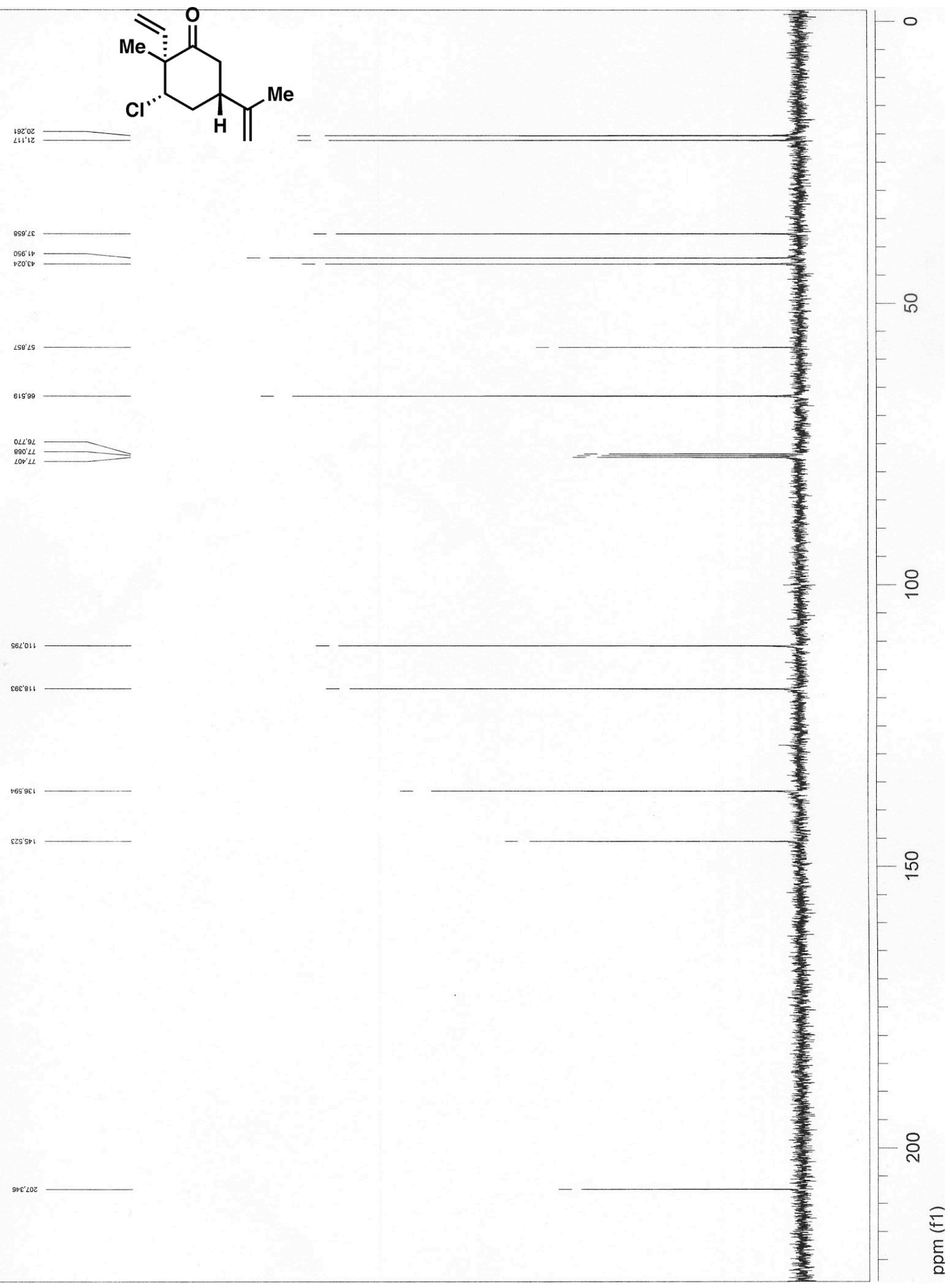




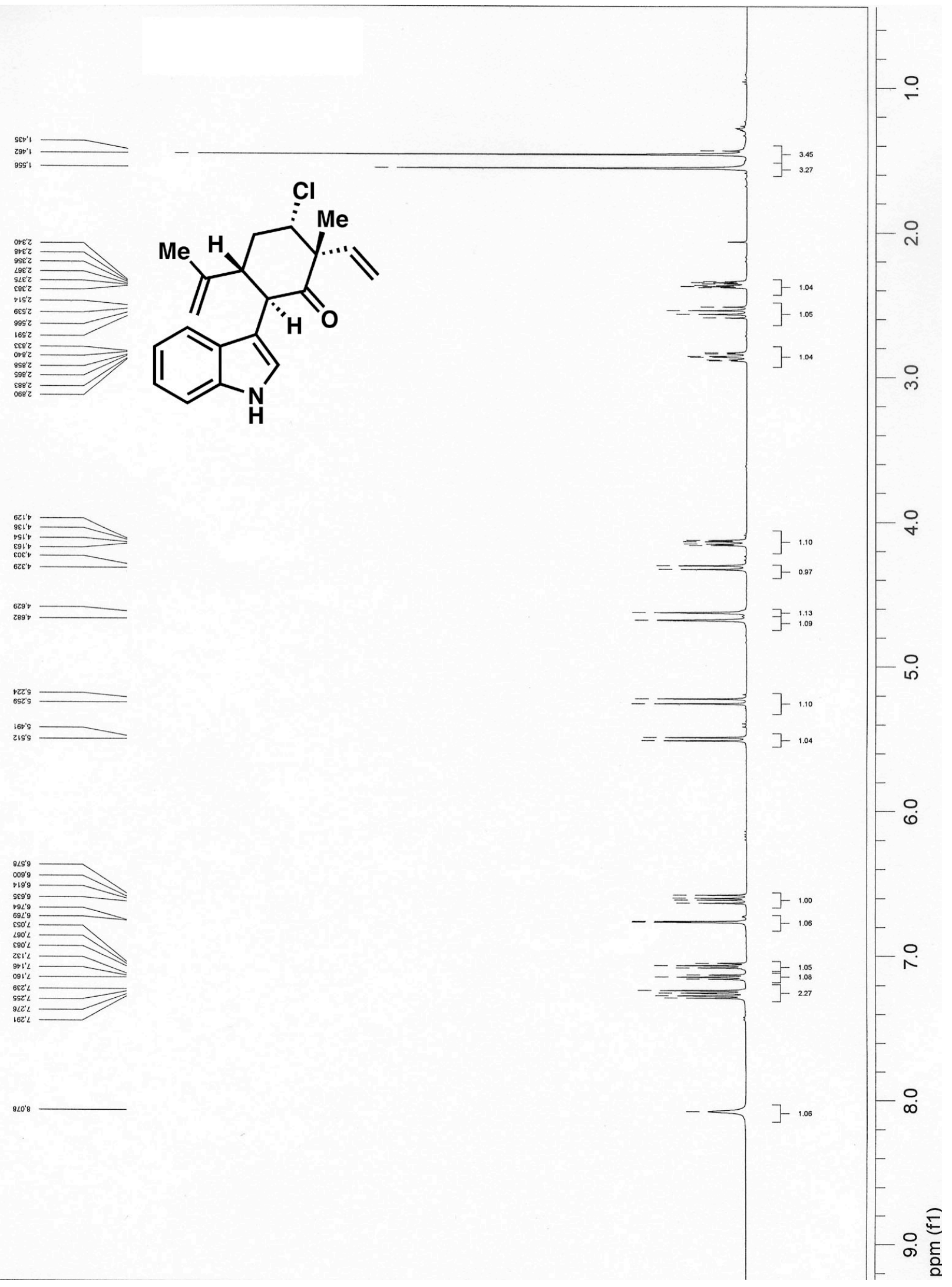




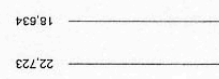

$\varepsilon \angle S^{\prime} 6 \varepsilon$

$20 e^{2} 8 t^{2}$

\begin{tabular}{l}
$168^{\prime} 85$ \\
$999^{\prime} 99$ \\
\hline
\end{tabular}

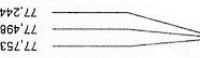

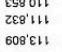

$\begin{aligned} & 210^{\prime} 611 \\ & 621611 \\ & 16 L^{\prime} 611\end{aligned}=$

8Lเ'zเ

soi'zzt

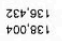

261'sนเ

$650^{\prime} 202$

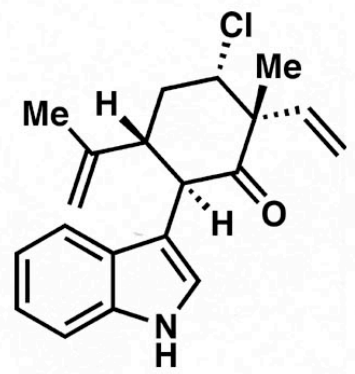

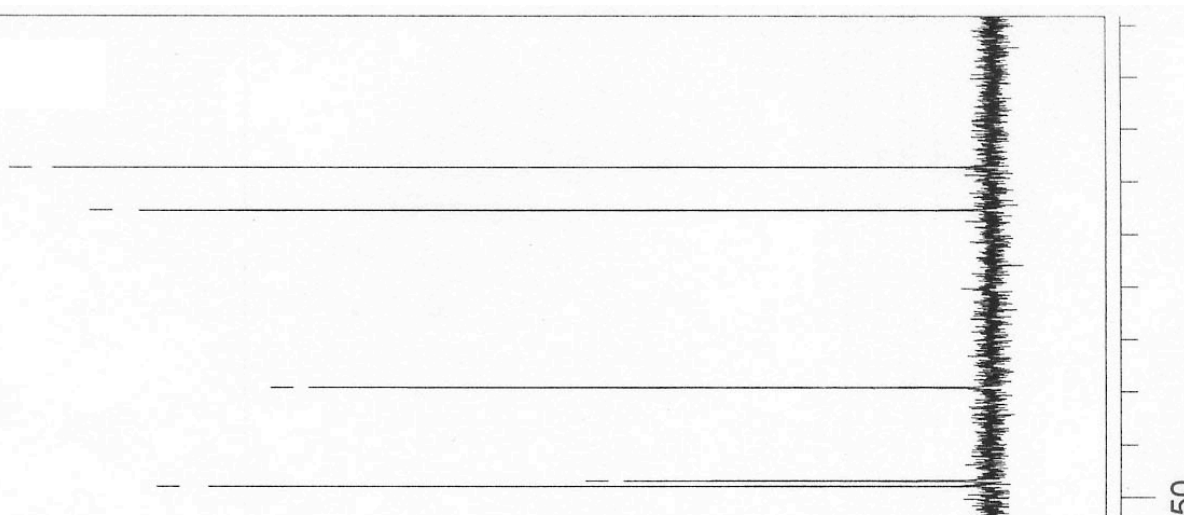

융

음

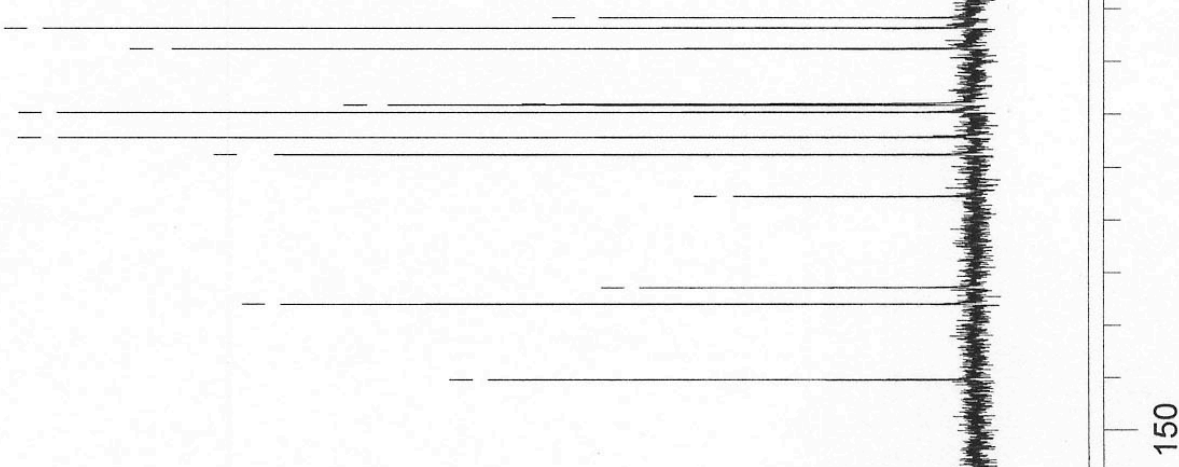

오 


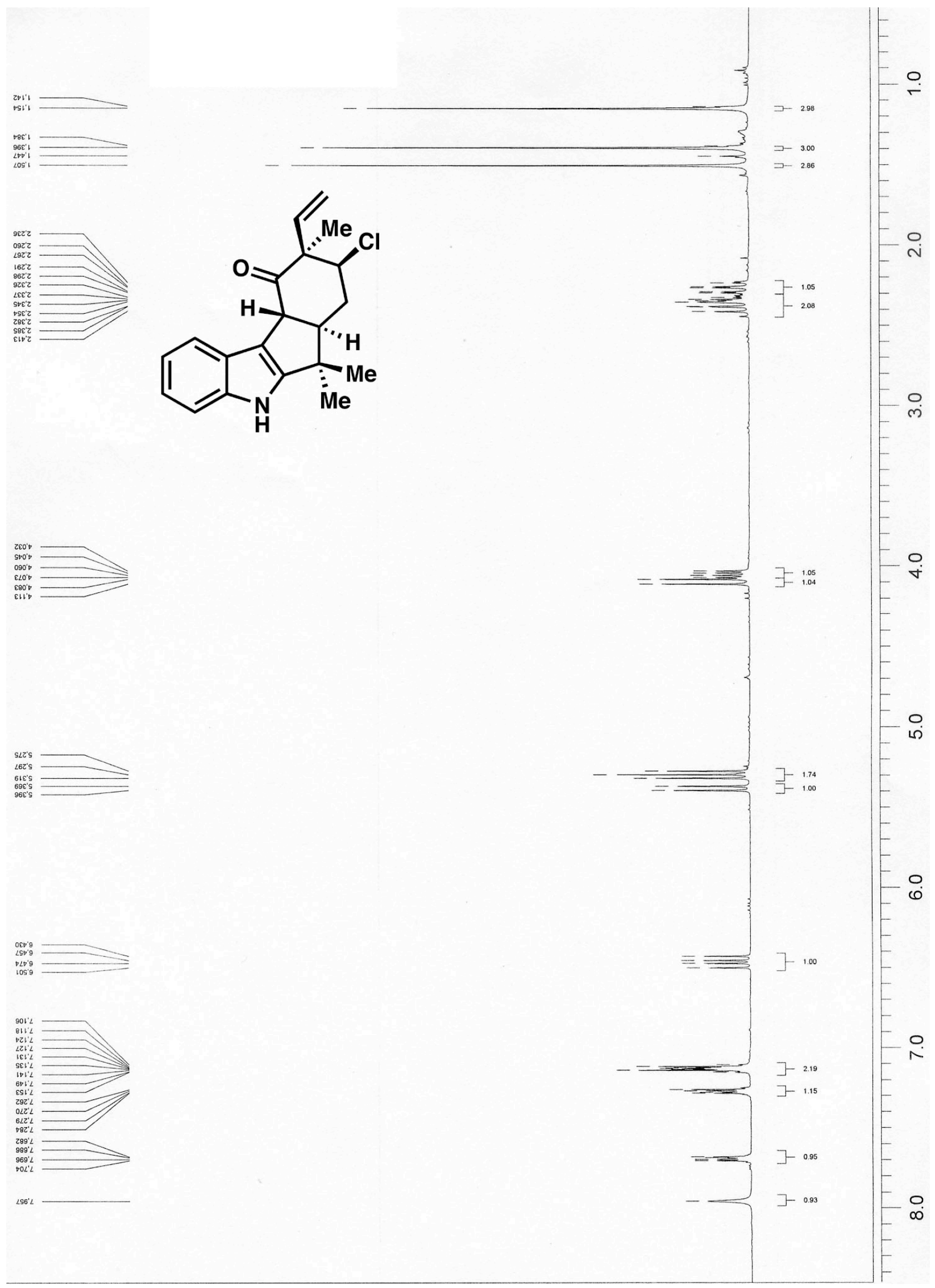



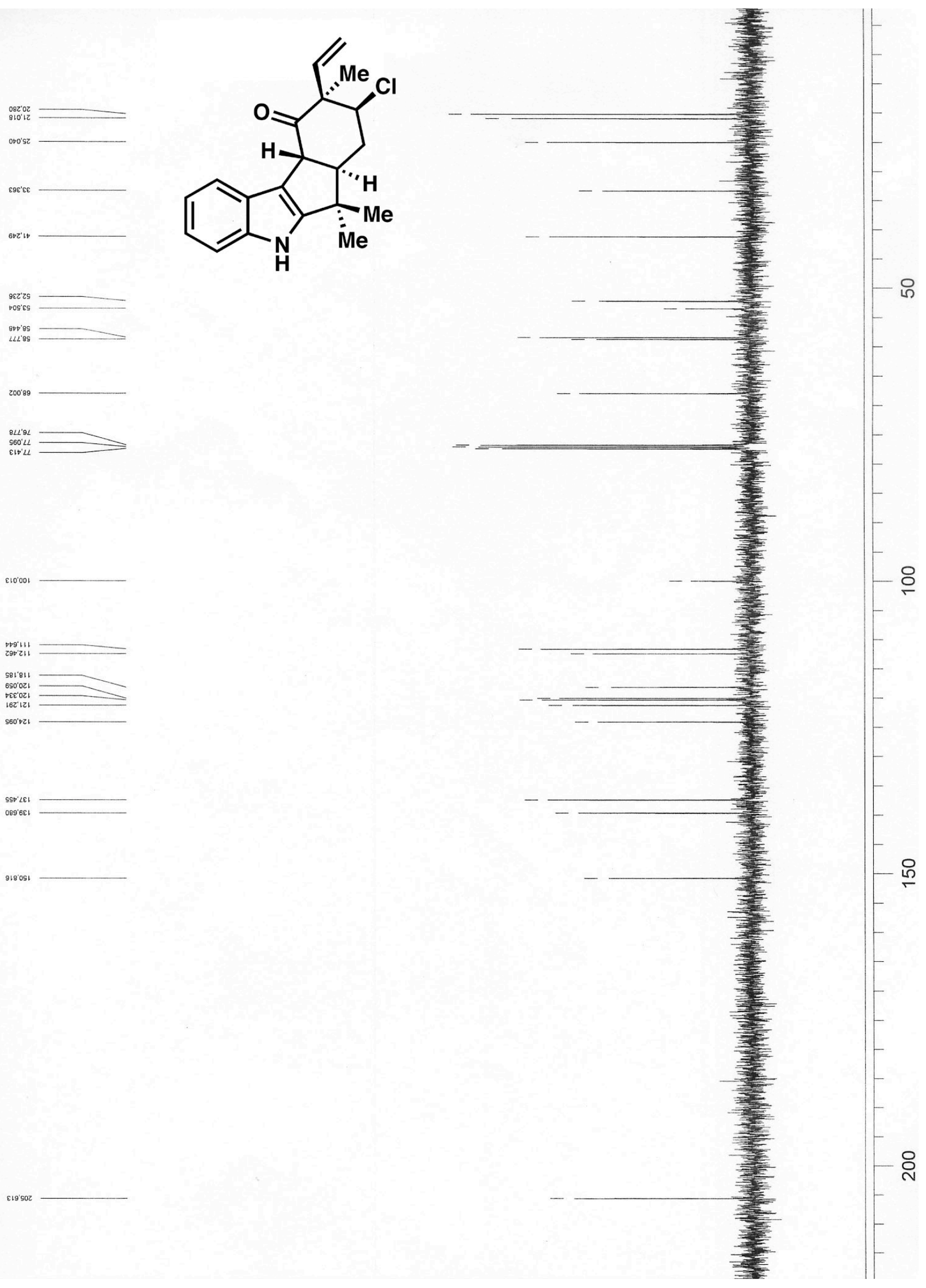


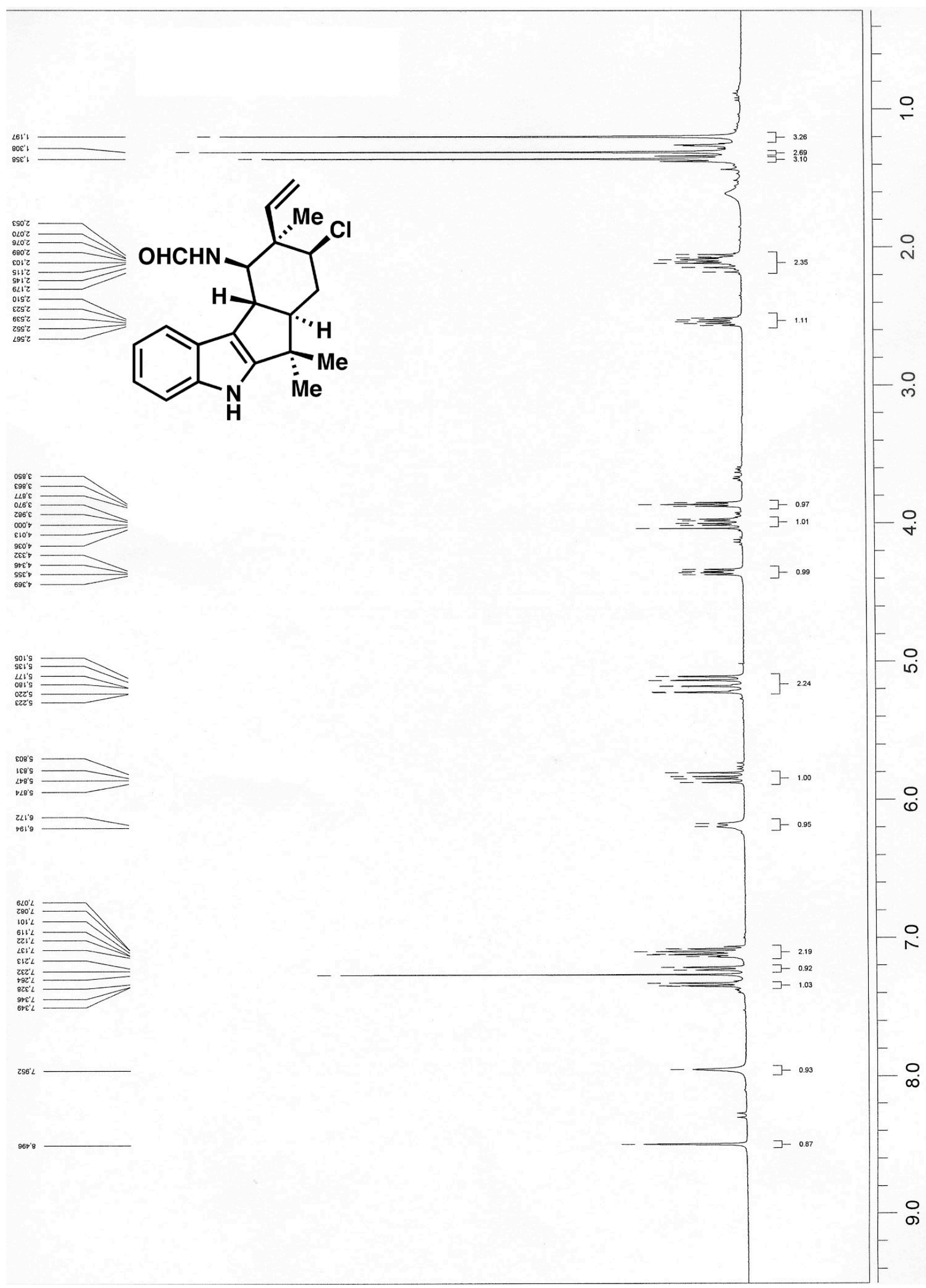




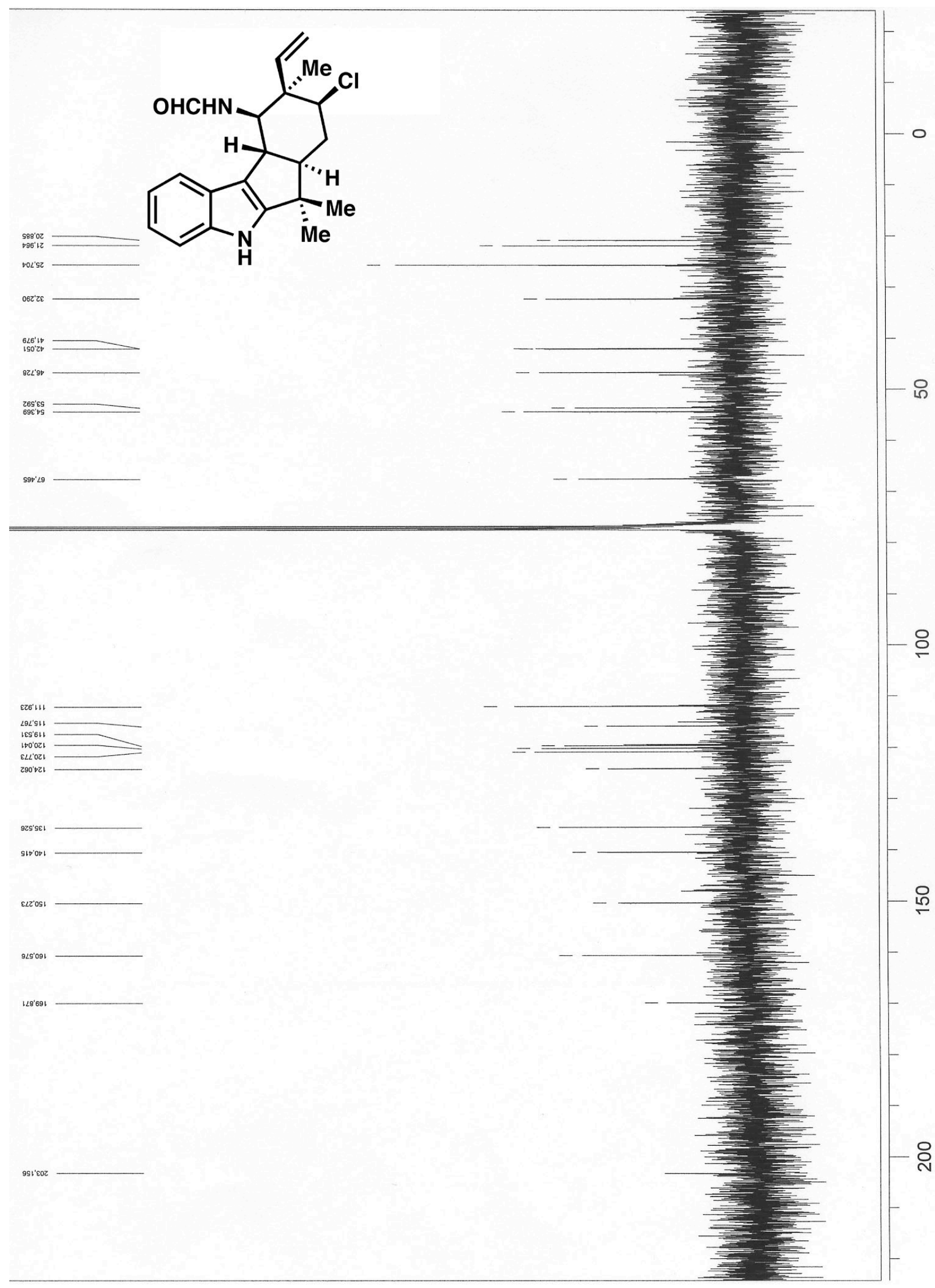




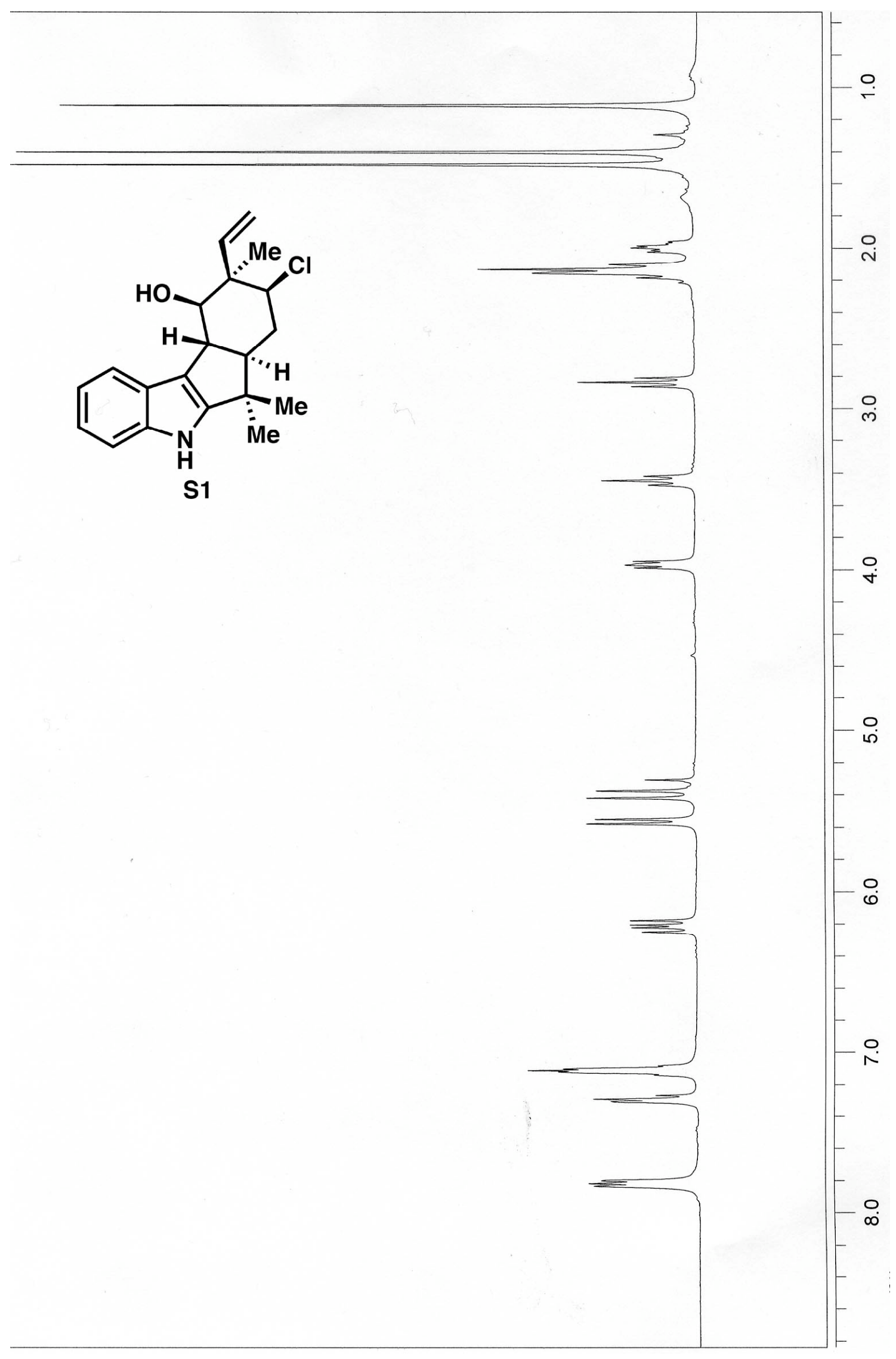


J. Am. Chem. Soc.

S-25

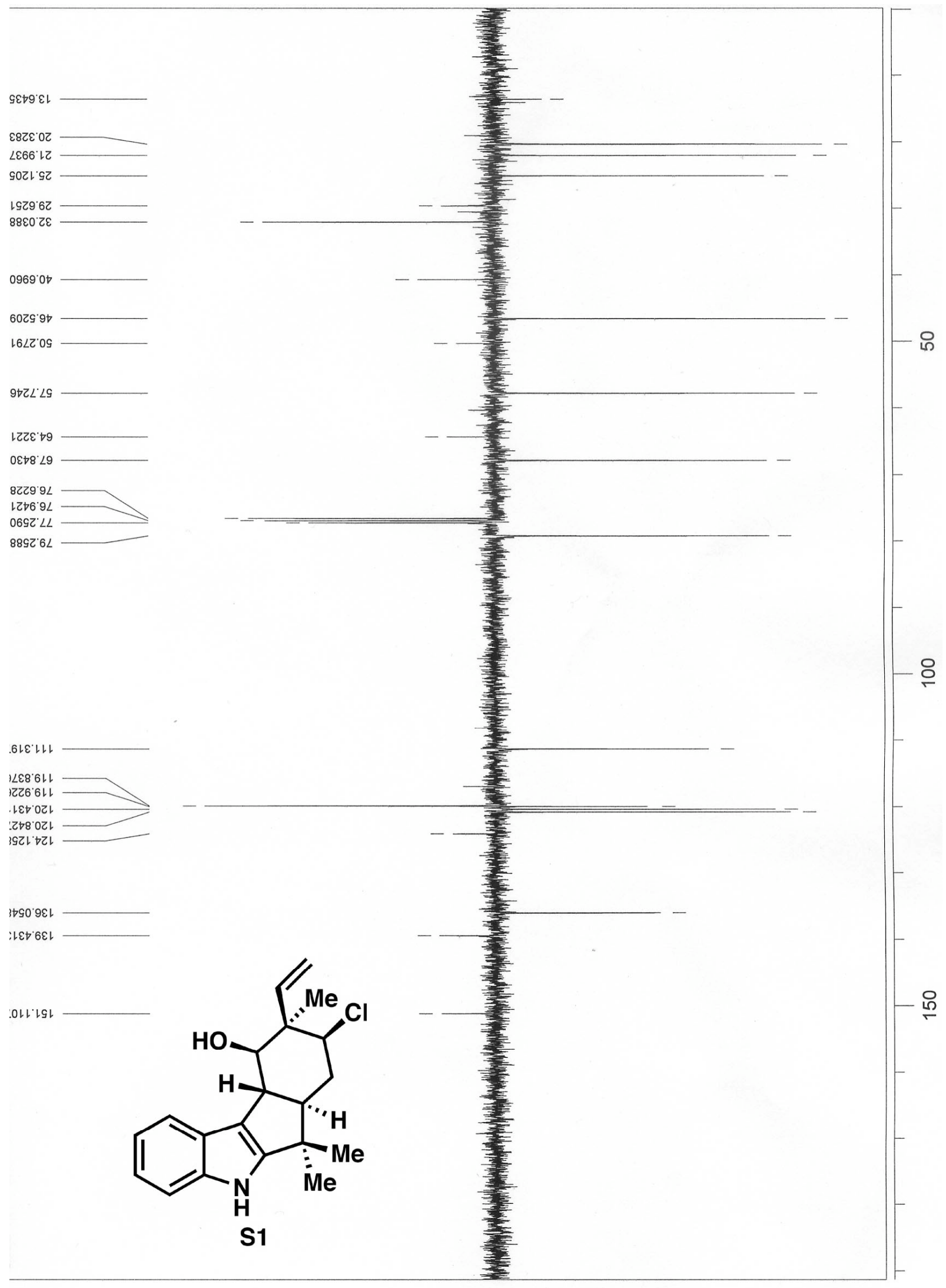




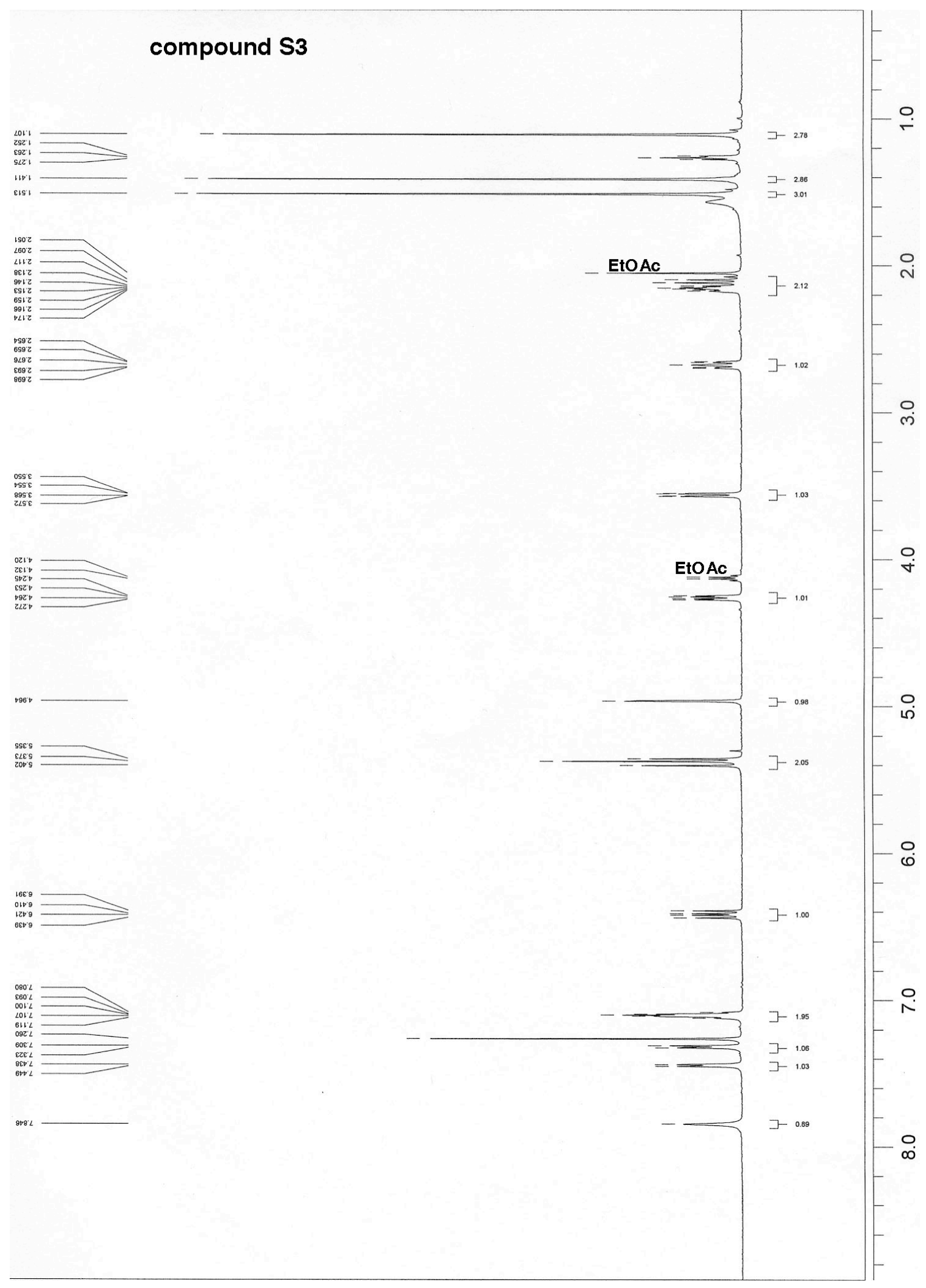




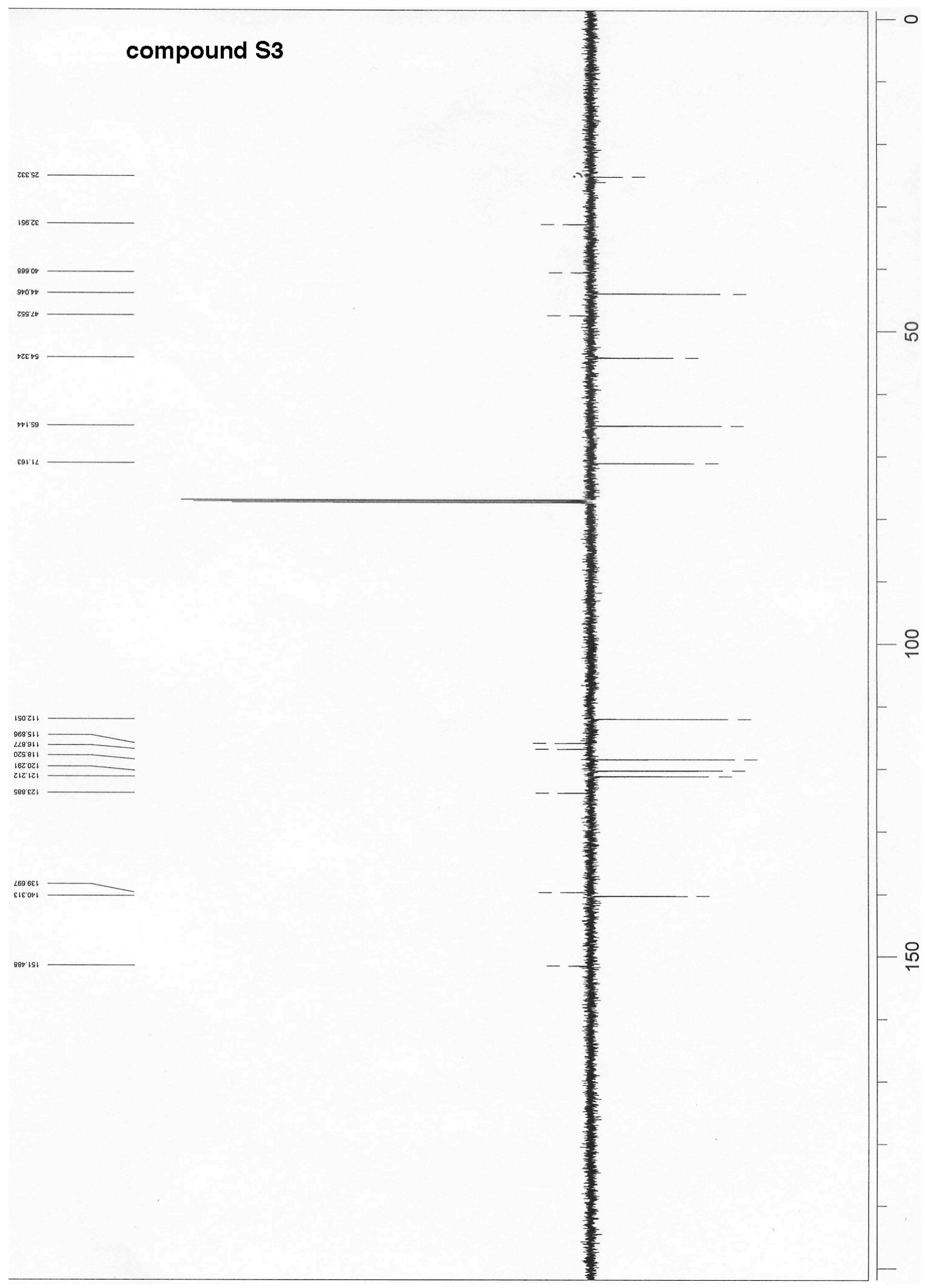



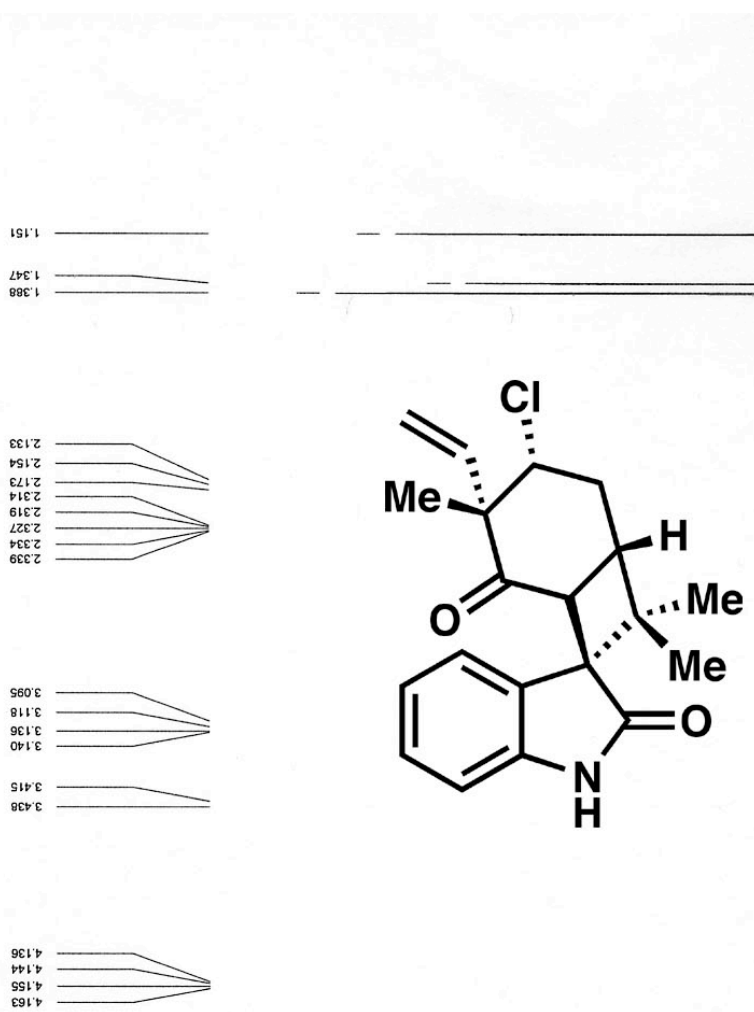

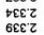

${ }_{9000}^{200 \% 5}$

$98 \mathrm{~g} ' \mathrm{~s}$
t9z's

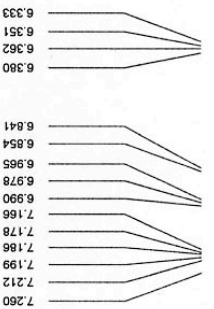

L8LL

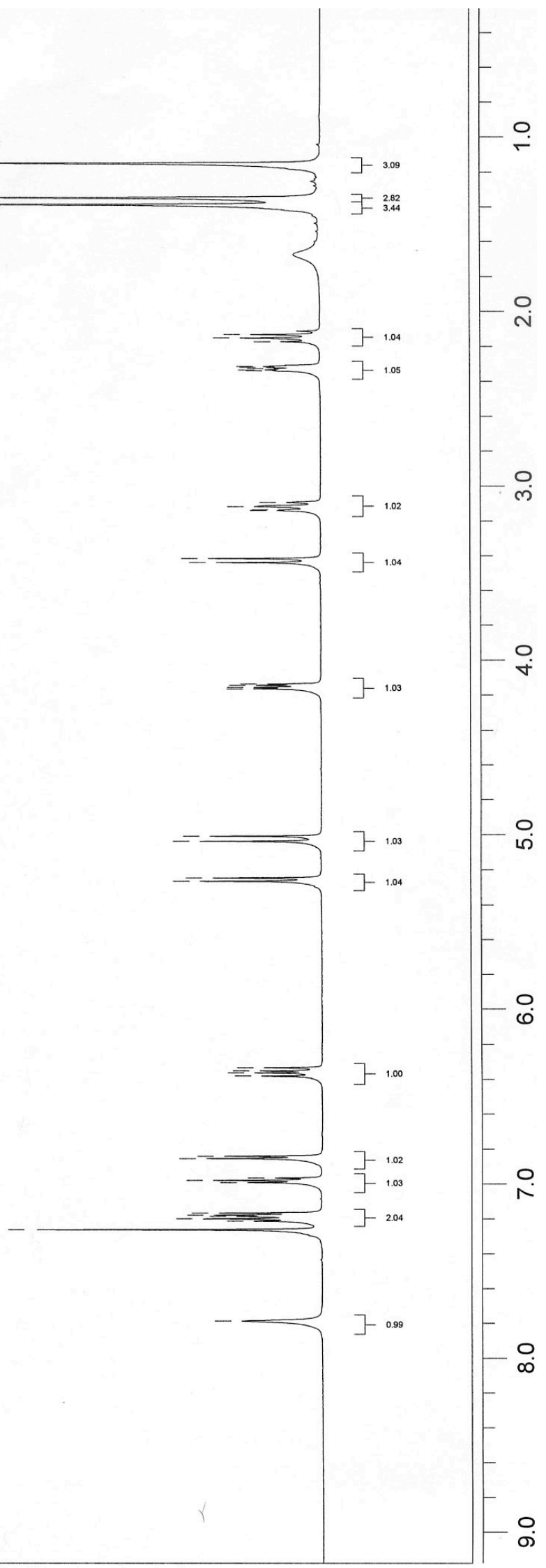



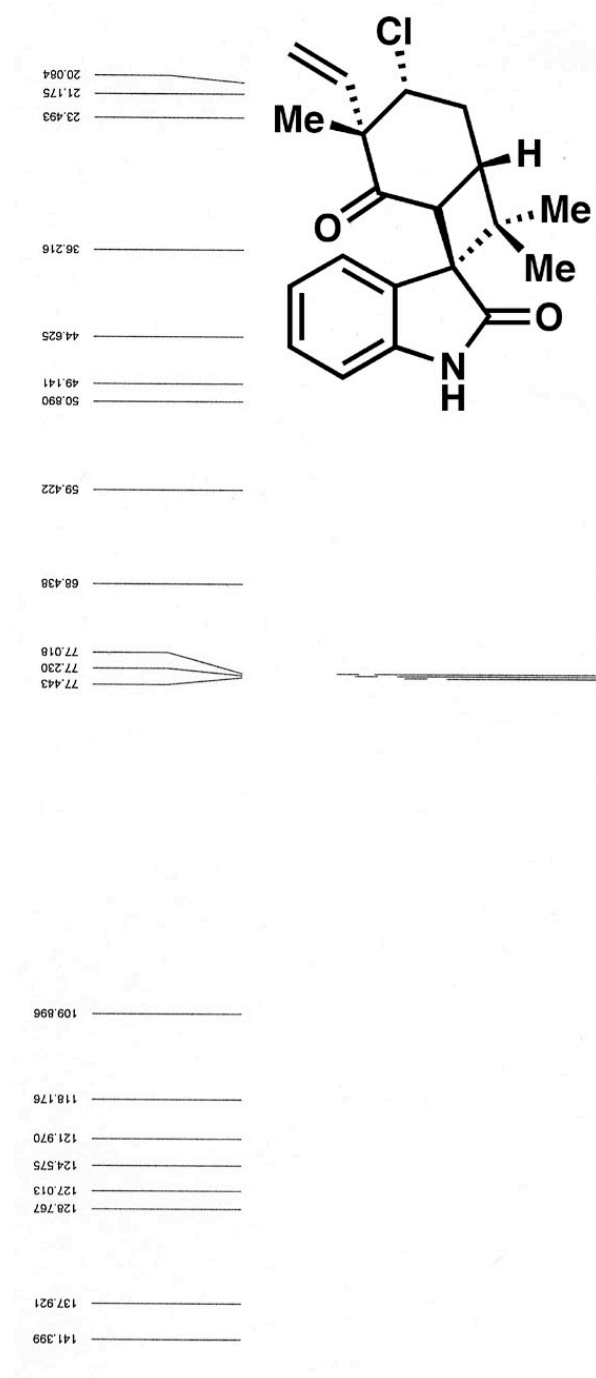

$66 \varepsilon^{\prime} \mathrm{W}$
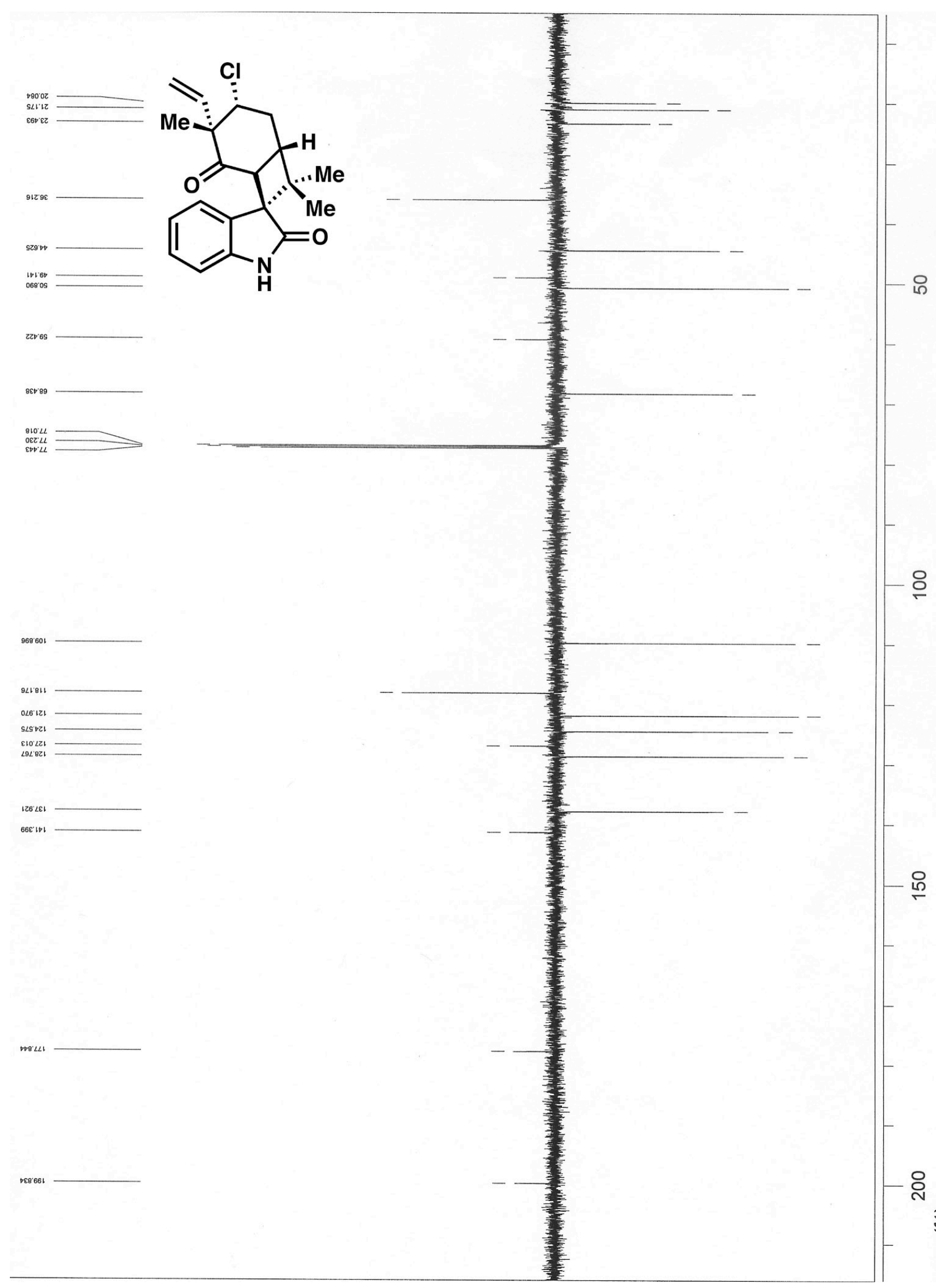

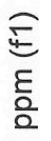




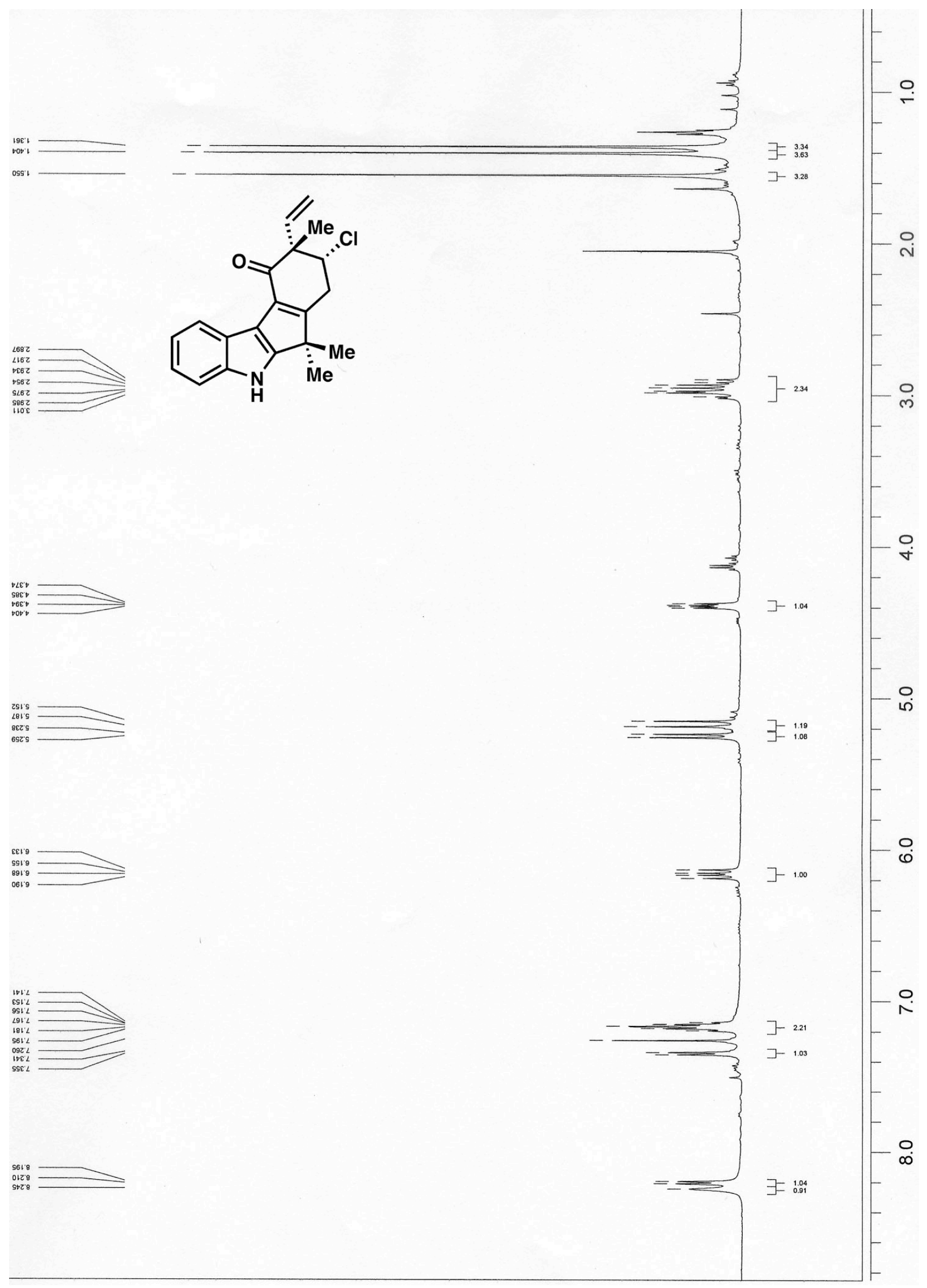



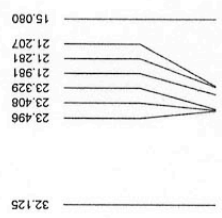

$\varepsilon 60^{\circ} \angle t$
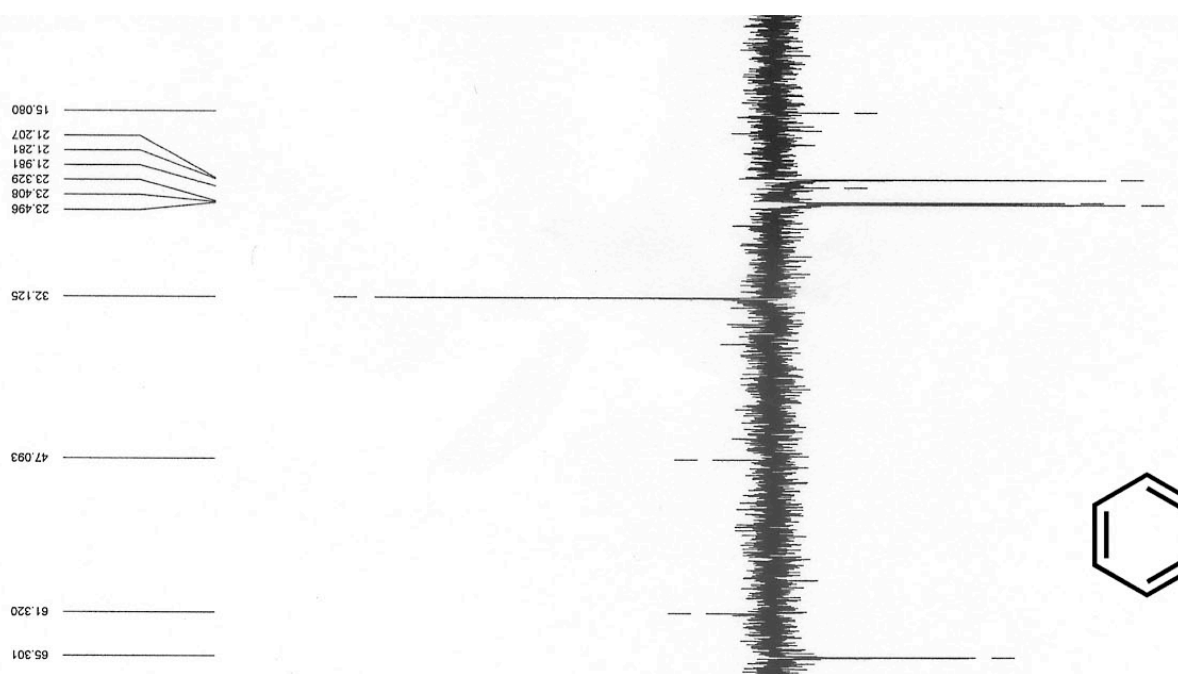

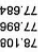

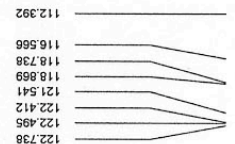

Lot't\&\&

80< 0 or 1

sitest

69069.

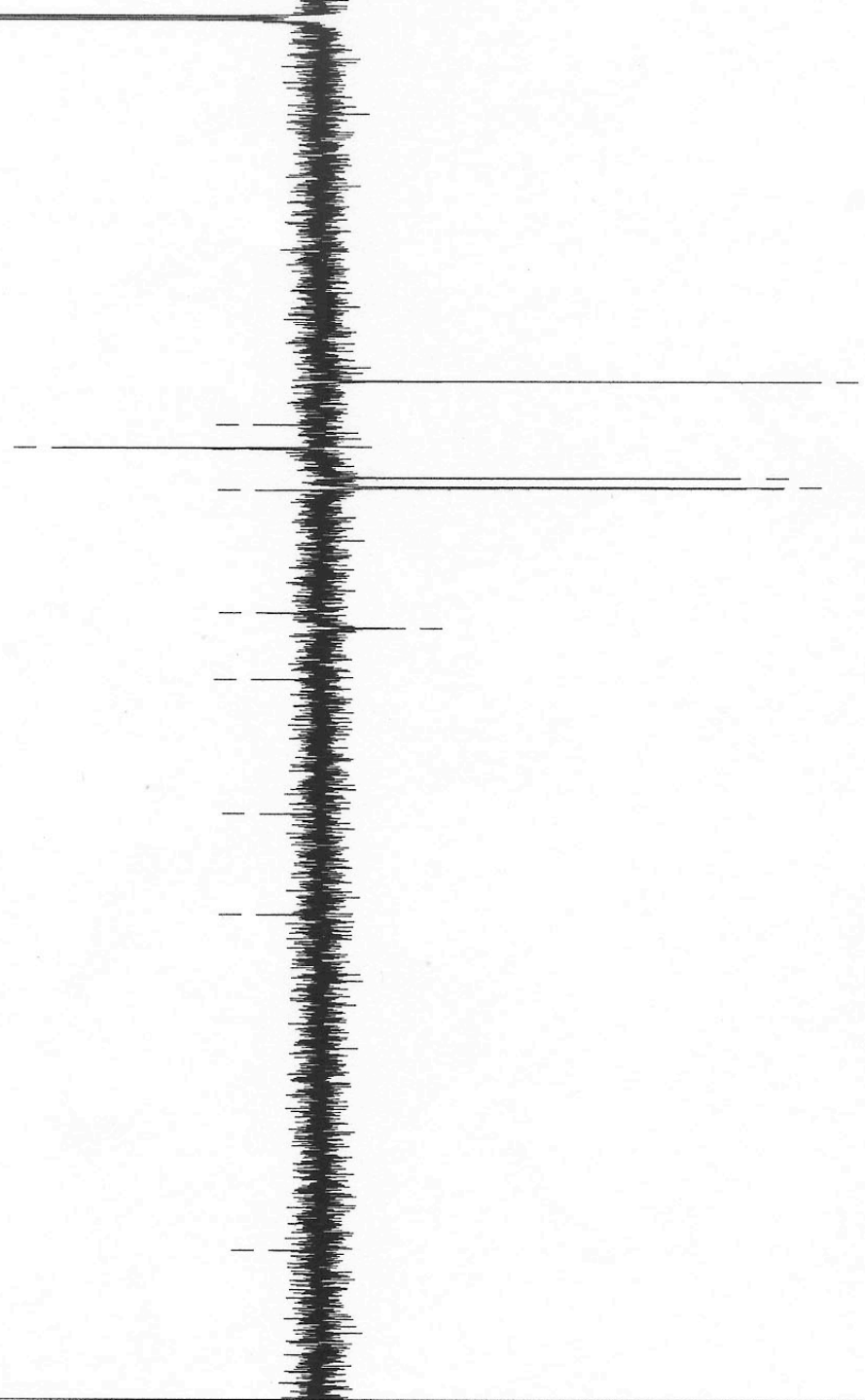




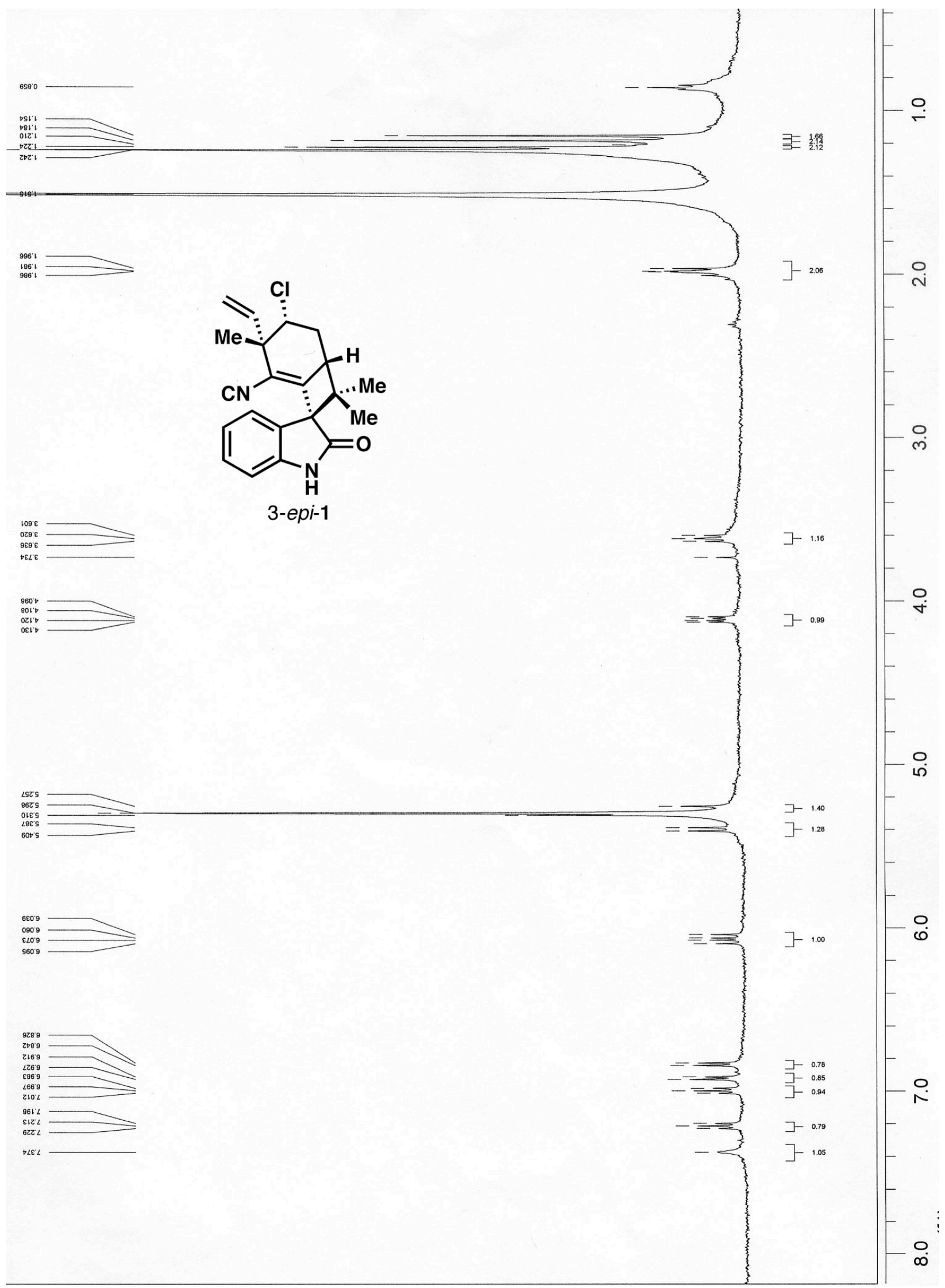



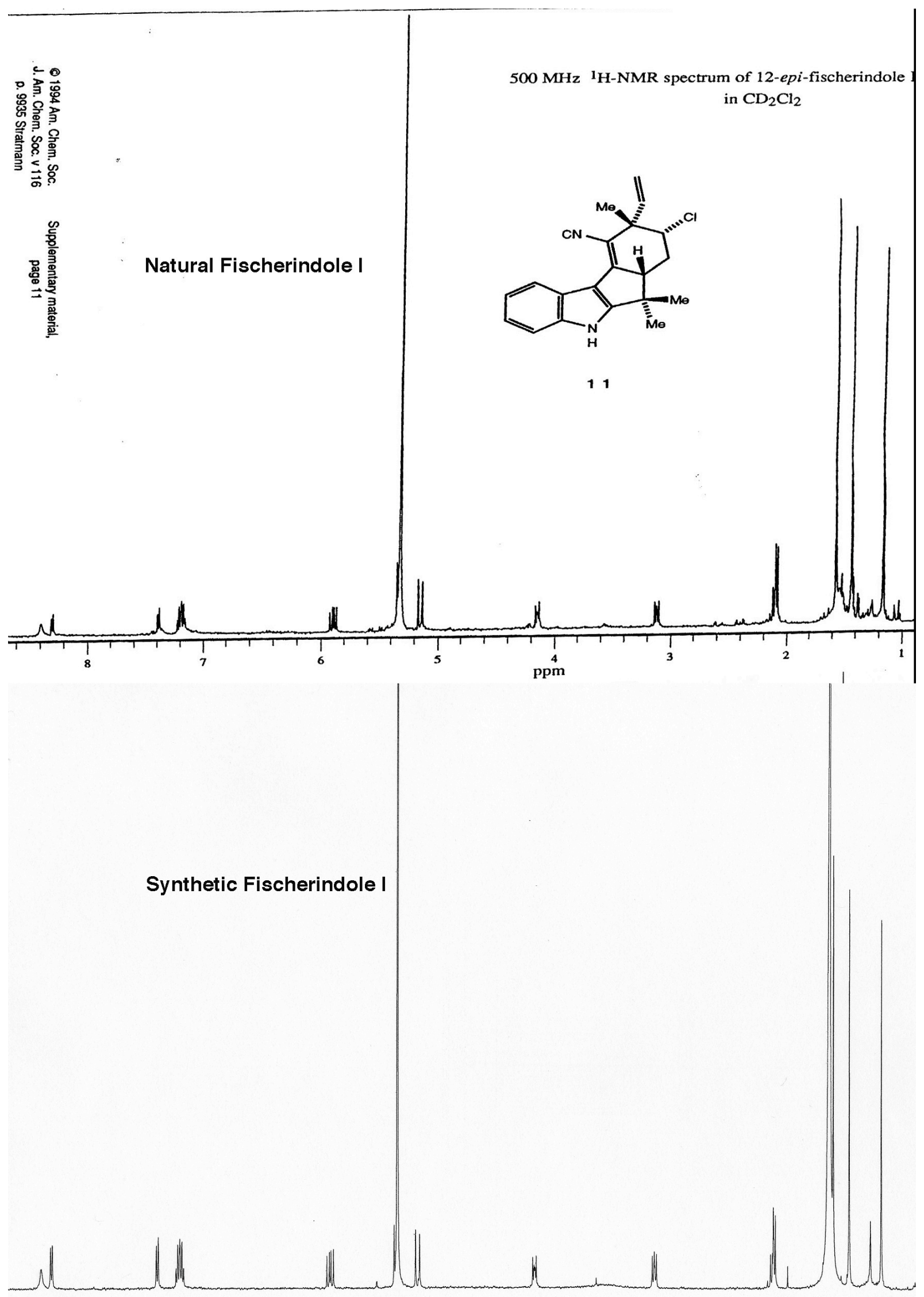


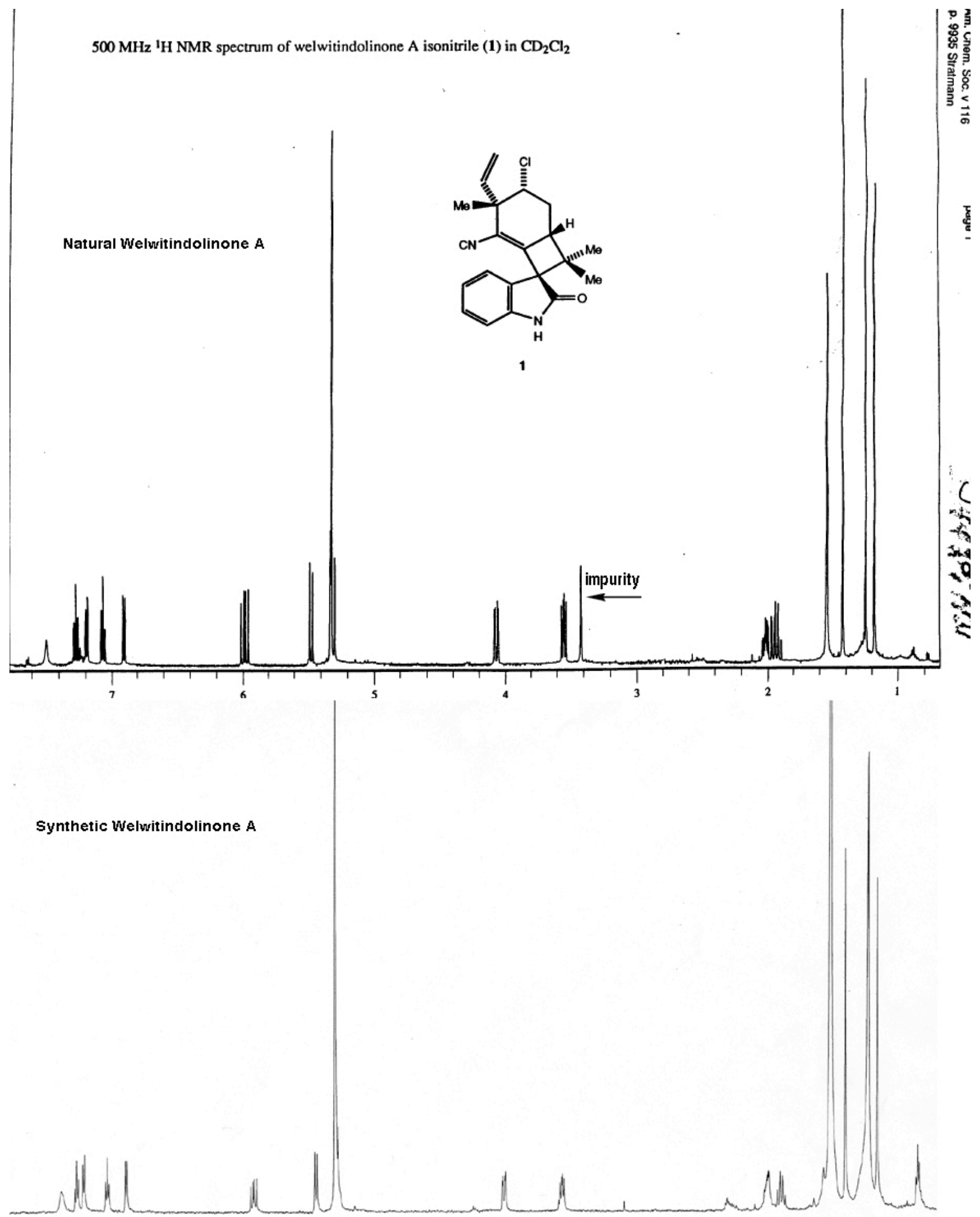




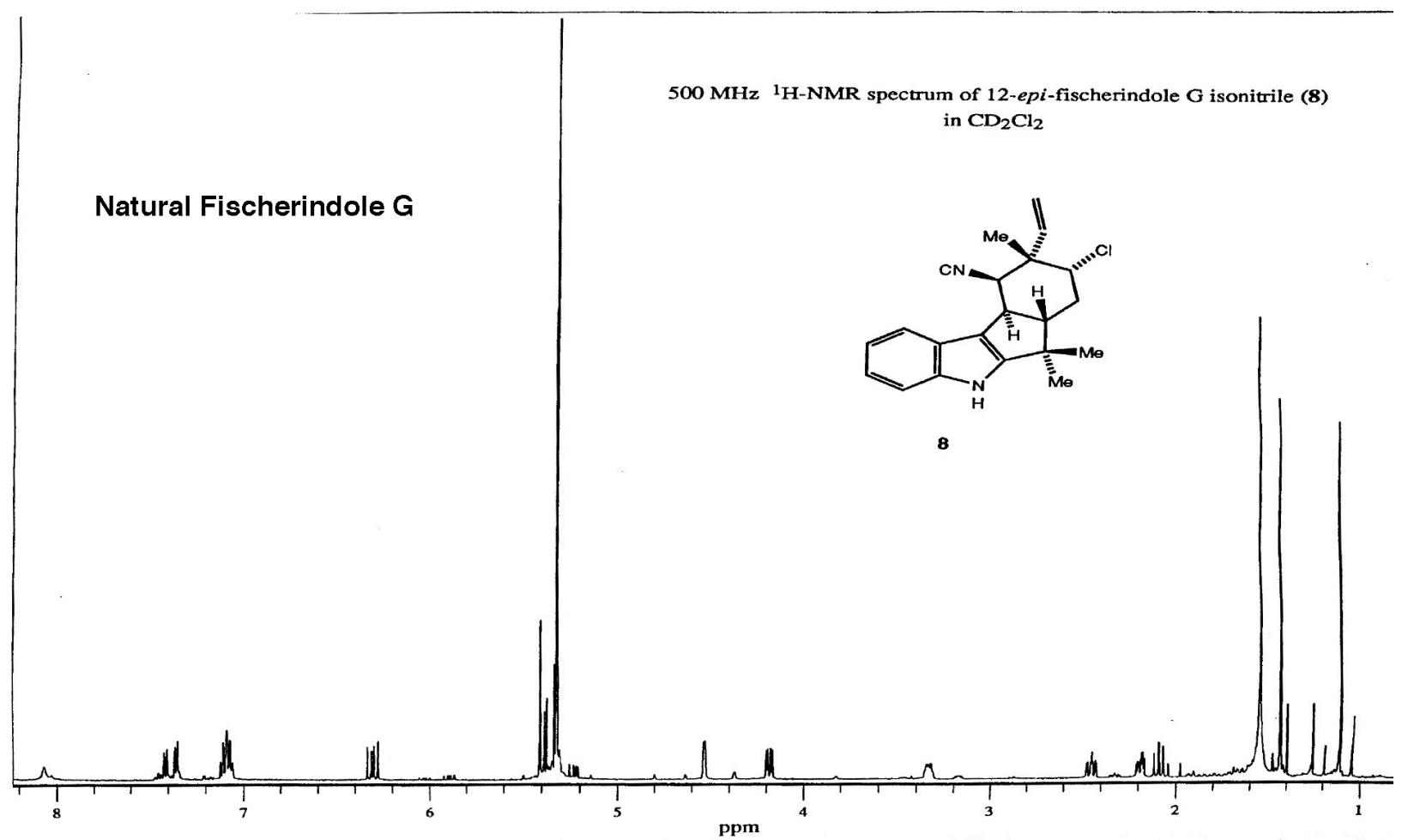

Synthetic Fischerindole G
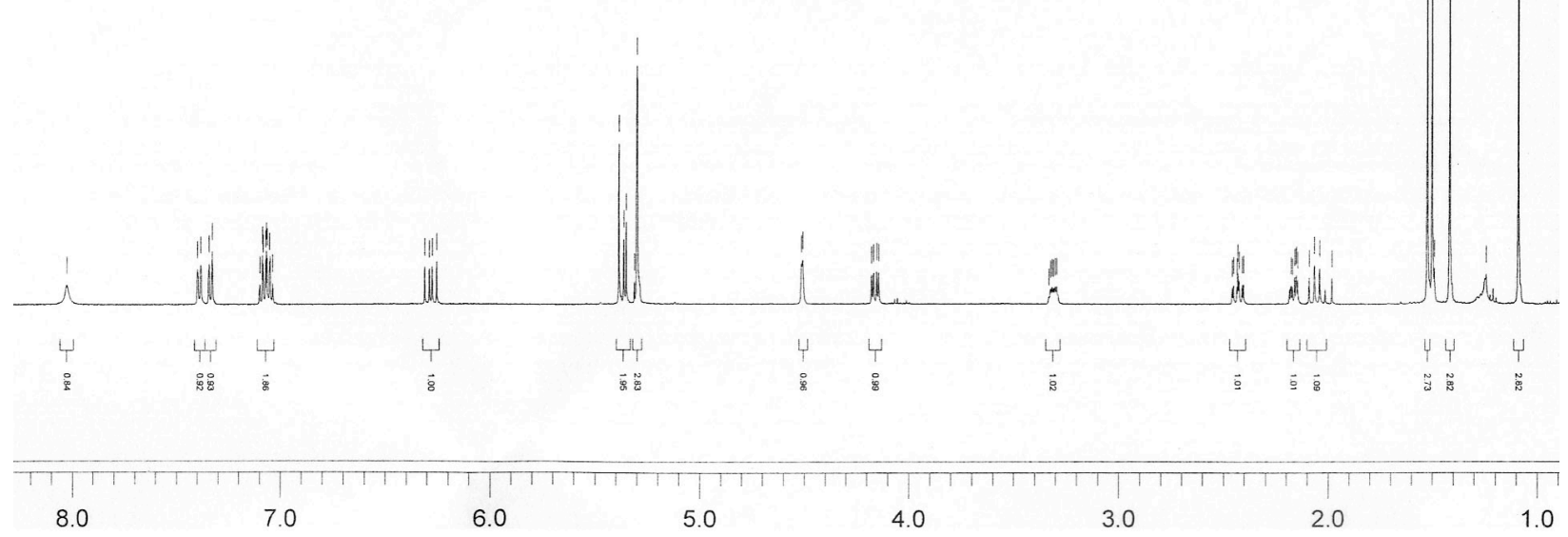